\title{
SIGN-CHANGING SOLUTIONS OF THE NONLINEAR HEAT EQUATION WITH PERSISTENT SINGULARITIES ${ }^{*, * *, * * *}$
}

\author{
Thierry Cazenave ${ }^{1, * * * *}$, Flávio Dickstein ${ }^{2}$, Ivan Naumkin ${ }^{3}$ \\ AND FRED B. WEISSLER ${ }^{4}$
}

\begin{abstract}
We study the existence of sign-changing solutions to the nonlinear heat equation $\partial_{t} u=$ $\Delta u+|u|^{\alpha} u$ on $\mathbb{R}^{N}, N \geq 3$, with $\frac{2}{N-2}<\alpha<\alpha_{0}$, where $\alpha_{0}=\frac{4}{N-4+2 \sqrt{N-1}} \in\left(\frac{2}{N-2}, \frac{4}{N-2}\right)$, which are singular at $x=0$ on an interval of time. In particular, for certain $\mu>0$ that can be arbitrarily large, we prove that for any $u_{0} \in \mathrm{L}_{\text {loc }}^{\infty}\left(\mathbb{R}^{N} \backslash\{0\}\right)$ which is bounded at infinity and equals $\mu|x|^{-\frac{2}{\alpha}}$ in a neighborhood of 0 , there exists a local (in time) solution $u$ of the nonlinear heat equation with initial value $u_{0}$, which is sign-changing, bounded at infinity and has the singularity $\beta|x|^{-\frac{2}{\alpha}}$ at the origin in the sense that for $t>0,|x|^{\frac{2}{\alpha}} u(t, x) \rightarrow \beta$ as $|x| \rightarrow 0$, where $\beta=\frac{2}{\alpha}\left(N-2-\frac{2}{\alpha}\right)$. These solutions in general are neither stationary nor self-similar.
\end{abstract}

Mathematics Subject Classification. 35K91, 35K58, 35C06, 35K67, 35A01, 35A21.

Received June 29, 2020. Accepted November 18, 2020.

Dedicated to Enrique Zuazua on the occasion of his 60th birthday.

\section{INTRODUCTION}

In this paper, we study the nonlinear heat equation

$$
\partial_{t} u=\Delta u+|u|^{\alpha} u
$$

\footnotetext{
* Research supported by the "Brazilian-French Network in Mathematics".

** Flavio Dickstein was partially supported by CNPq (Brasil).

*** Ivan Naumkin is a Fellow of Sistema Nacional de Investigadores. He was partially supported by project PAPIIT IA101820.

Keywords and phrases: Nonlinear heat equation, sign-changing solutions, singular self-similar solutions, singular stationary solutions, persistent singularities.

${ }^{1}$ Sorbonne Université, CNRS, Université de Paris, Laboratoire Jacques-Louis Lions, B.C. 187, 4 place Jussieu, 75252 Paris Cedex 05, France.

2 Instituto de Matemática, Universidade Federal do Rio de Janeiro, Caixa Postal 68530, 21944-970 Rio de Janeiro, RJ, Brazil.

3 Departamento de Física Matemática, Instituto de Investigaciones en Matemáticas Aplicadas y en Sistemas, Universidad Nacional Autónoma de México, Apartado Postal 20-126, Ciudad de México 01000, Mexico.

4 Université Sorbonne Paris Nord, CNRS UMR 7539 LAGA, 99 Avenue J.-B. Clément, 93430 Villetaneuse, France.

**** Corresponding author: thierry.cazenave@sorbonne-universite.fr
} 
on $\mathbb{R}^{N}$ with

$$
N \geq 3 \text { and } \frac{2}{N-2}<\alpha<\alpha_{0}
$$

where

$$
\alpha_{0}=\frac{4}{N-4+2 \sqrt{N-1}} .
$$

(Note that $\frac{2}{N-2}<\alpha_{0}<\frac{4}{N-2}$, see Lem. 2.1 (i).) We are interested in sign-changing solutions of (1.1) which have a singularity at $x=0$ on an interval of time.

Positive solutions of (1.1) with a standing or moving singularity have been well studied. The simplest, for all $\alpha>\frac{2}{N-2}$, is the homogeneous stationary solution $\beta^{\frac{1}{\alpha}}|x|^{-\frac{2}{\alpha}}$ where

$$
\beta=\frac{2}{\alpha}\left(N-2-\frac{2}{\alpha}\right)>0 .
$$

Moreover, for all $\frac{2}{N-2}<\alpha<\frac{4}{N-2}$, (1.1) has a one-parameter family $\left(U_{\lambda}\right)_{\lambda>0} \subset \mathrm{C}^{2}\left(\mathbb{R}^{N} \backslash\{0\}\right)$ of radially symmetric, positive, singular stationary solutions satisfying $|x|^{\frac{2}{\alpha}} U_{\lambda}(x) \rightarrow \beta^{\frac{1}{\alpha}}$ as $x \rightarrow 0$ and $|x|^{N-2} U_{\lambda}(x) \rightarrow \lambda$ as $|x| \rightarrow \infty$. Furthermore, for this range of $\alpha$, the family $\left(U_{\lambda}\right)_{\lambda>0}$ and $\beta^{\frac{1}{\alpha}}|x|^{-\frac{2}{\alpha}}$ constitute all the positive, radially symmetric, singular stationary solutions of (1.1). See ([21], Prop. 3.1). Under the stronger assumption (1.2), these solutions can be used as prototypes to construct positive solutions of (1.1) with a moving singularity, i.e. a singularity located at $x=\xi(t)$ for every $t$ in some interval, under appropriate conditions on the function $\xi(\cdot)$. See $[17,20]$. Positive self-similar solutions of $(1.1)$, both forward and backward, with a standing or moving singularity, have also been constructed, see $[18,19]$. The finite-time blowup and the long-time asymptotic behavior of positive singular solutions of $(1.1)$ are studied in $[7,16]$.

Sign changing stationary solutions of equation (1.1) have been less studied. We show here that for all $\frac{2}{N-2}<$ $\alpha<\frac{4}{N-2}$, equation (1.1) has sign-changing, radially symmetric, stationary solutions that are singular at $x=0$. These solutions behave like $\beta^{\frac{1}{\alpha}}|x|^{-\frac{2}{\alpha}}$ at the origin and oscillate indefinitely as $|x| \rightarrow \infty$. See Proposition 6.1 and Corollary 6.2.

For the same range $\frac{2}{N-2}<\alpha<\frac{4}{N-2}$, equation (1.1) also has sign-changing, radially symmetric, self-similar solutions which are singular for all positive time. More precisely, it follows from ([5], Thm. 1.3) that there exist an integer $\bar{m} \geq 0$ and an increasing sequence $\left(\mu_{m}\right)_{m \geq \bar{m}} \subset(0, \infty), \mu_{m} \rightarrow \infty$ as $m \rightarrow \infty$, such that for each $\mu_{m}$ there exists a radially symmetric self-similar solution

$$
U(t, x)=t^{-\frac{1}{\alpha}} f\left(\frac{|x|}{\sqrt{t}}\right)
$$

of (1.1) in the sense of distributions, where the profile $f \in \mathrm{C}^{2}(0, \infty)$ has exactly $m$ zeros and satisfies

$$
f^{\prime \prime}+\left(\frac{N-1}{r}+\frac{r}{2}\right) f^{\prime}+\frac{1}{\alpha} f+|f|^{\alpha} f=0
$$

Moreover, $r^{\frac{2}{\alpha}} f(r) \rightarrow \beta^{\frac{1}{\alpha}}$ as $r \rightarrow 0$ and $r^{\frac{2}{\alpha}} f(r) \rightarrow(-1)^{m} \mu_{m}$ as $r \rightarrow \infty$. It follows that $|x|^{\frac{2}{\alpha}} U(t, x) \rightarrow \beta^{\frac{1}{\alpha}}$ as $|x| \rightarrow 0$ for all $t>0$, and $U(t, \cdot) \rightarrow(-1)^{m} \mu_{m}|\cdot|^{-\frac{2}{\alpha}}$ in $\mathrm{L}_{\text {loc }}^{1}\left(\mathbb{R}^{N}\right)$ as $t \rightarrow 0$.

The purpose of this article is to construct sign-changing solutions of (1.1) which are singular at $x=0$ for small positive time, and which are neither stationary nor self-similar. The construction of these solutions is based on the following perturbation result. 
Theorem 1.1. Assume (1.2). Let $S>0$ and

$$
U \in \mathrm{L}_{\text {loc }}^{\alpha+1}\left((0, S) \times \mathbb{R}^{N}\right) \cap \mathrm{C}\left((0, S) \times\left(\mathbb{R}^{N} \backslash\{0\}\right)\right)
$$

satisfy for some constant $C$

$$
\left.|| x\right|^{\frac{2}{\alpha}} U(t, x)-\beta^{\frac{1}{\alpha}} \mid \leq C\left[\left(\frac{|x|}{|x|+\sqrt{t}}\right)^{\rho}+|x|^{\frac{2}{\alpha}}\right]
$$

for $0<t<S$ and $x \neq 0$, where

$$
\rho=\frac{2}{\alpha}-\frac{N-2}{2}-\sqrt{\frac{(N-2)^{2}}{4}-\beta(\alpha+1)}
$$

and $\beta$ is given by (1.4). ( $\rho>0$ by Lemma 2.1 (iii) below.) Assume further that there exists $U_{0} \in \mathrm{L}_{\mathrm{loc}}^{1}\left(\mathbb{R}^{N}\right)$ such that

$$
U(t, \cdot) \underset{t \rightarrow 0}{\longrightarrow} U_{0}(\cdot)
$$

in $\mathrm{L}_{\text {loc }}^{1}\left(\mathbb{R}^{N}\right)$. Given $\delta>0$ and $u_{0} \in \mathrm{L}_{\text {loc }}^{1}\left(\mathbb{R}^{N}\right) \cap \mathrm{L}^{\infty}(\{|x|>\delta\})$ such that

$$
u_{0}(x)=U_{0}(x) \text { a.e. on }\{|x|<\delta\},
$$

there exist $T \in(0, S)$ and a solution $u \in \mathrm{L}_{\text {loc }}^{\alpha+1}\left((0, T) \times \mathbb{R}^{N}\right) \cap \mathrm{C}\left((0, T) \times\left(\mathbb{R}^{N} \backslash\{0\}\right)\right)$ of

$$
\partial_{t} u-\Delta u=|u|^{\alpha} u-\left[\partial_{t} U-\Delta U-|U|^{\alpha} U\right],
$$

in the sense of distributions $\mathcal{D}^{\prime}\left((0, T) \times \mathbb{R}^{N}\right)$, such that

$$
u(t) \underset{t \rightarrow 0}{\longrightarrow} u_{0} \quad \text { in } \mathrm{L}_{\mathrm{loc}}^{1}\left(\mathbb{R}^{N}\right)
$$

and

$$
|u(t, x)-U(t, x)| \leq C\left(1+|x|^{-\eta}\right), \quad 0<t<T, x \neq 0,
$$

where

$$
\eta=\frac{N-2}{2}-\sqrt{\frac{(N-2)^{2}}{4}-\beta(\alpha+1)}>0 .
$$

In Theorem 1.1, the choice of $u_{0}$ is both flexible and rigid. On the one hand, for $|x|>\delta$ there is complete freedom to choose $u_{0}$ as long as it is bounded. On the other hand, for $|x|<\delta, u_{0}$ must agree precisely with $U_{0}$, the initial value of the given function $U$. The following remark gives information on the relationship between $U$ and $u$, and how $u_{0}$ affects this relationship.

Remark 1.2. (i) In Theorem 1.1, $U$ need not be a solution of (1.1), so that $u$ need not be a solution of (1.1). However, if $U$ solves (1.1), then so does $u$.

(ii) In Theorem 1.1, $U$ need not be radially symmetric. Even if $U$ is radially symmetric, $u$ is not radially symmetric if $u_{0}$ is not. (Recall that $u_{0}$ is specified only in the ball of radius $\delta$.)

(iii) The function $u$ cannot be a stationary solution of (1.1), unless $u_{0}$ is a stationary solution of (1.1), by (1.11). 
(iv) The function $u$ cannot be a self-similar solution of (1.1), unless $u_{0}$ is homogeneous. Indeed, recall that the initial value of a self-similar solution, if it exists, is always homogeneous.

(v) Since $\eta<\frac{N-2}{2}<\frac{2}{\alpha}$ by (1.2) and (2.2), it follows from (1.7) and (1.12) that $u$ given by Theorem 1.1 is singular for all $0<t<T$ at $x=0$, and has the same singular behavior as $U(t)$, i.e.

$$
|x|^{\frac{2}{\alpha}} u(t, x) \underset{x \rightarrow 0}{\longrightarrow} \beta .
$$

In order to use Theorem 1.1 to construct sign-changing solutions of (1.1) with a singularity at $x=0$, we consider separately the cases where $U$ is a radially symmetric stationary solution of (1.1), and where $U$ is a radially symmetric self-similar solution of (1.1) with singular profile. This gives the following two theorems.

Theorem 1.3. Assume (1.2). Let $U$ be a radially symmetric, stationary solution of (1.1) that is singular at $x=0$. Given $\delta>0$ and $u_{0} \in \mathrm{L}_{\mathrm{loc}}^{1}\left(\mathbb{R}^{N}\right) \cap \mathrm{L}^{\infty}(\{|x|>\delta\})$ such that

$$
u_{0}(x)=U(x) \text { a.e. on }\{|x|<\delta\}
$$

there exist $T>0$ and a solution $u \in \mathrm{L}_{\text {loc }}^{\alpha+1}\left((0, T) \times \mathbb{R}^{N}\right) \cap \mathrm{C}\left((0, T) \times\left(\mathbb{R}^{N} \backslash\{0\}\right)\right)$ of (1.1) in the sense of distributions, such that (1.12) holds and $u(0)=u_{0}$ in the sense (1.11). Moreover, $u(t)$ is singular at $x=0$ for all $t<T$ and satisfies (1.14).

We stress the fact that there exist sign-changing stationary solutions $U$ to which Theorem 1.3 applies, by Corollary 6.2. In this case, if $\delta$ is sufficiently large, then the solution $u$ is sign-changing for small time by (1.11). See below for further discussion of this point.

Theorem 1.4. Assume (1.2). Let $f \in \mathrm{C}^{2}(0, \infty)$ be a solution of the equation (1.5) having the singularity $r^{\frac{2}{\alpha}} f(r) \rightarrow \beta^{\frac{1}{\alpha}}$ as $r \rightarrow 0$, let

$$
\mu=\lim _{r \rightarrow \infty} r^{\frac{2}{\alpha}} f(r) \in \mathbb{R}
$$

which exists by Proposition 7.2. Let

$$
U(t, x)=t^{-\frac{1}{\alpha}} f\left(\frac{|x|}{\sqrt{t}}\right), \quad t>0, x \neq 0,
$$

so that $U$ is a self-similar solution of (1.1) by Proposition 7.2. Given $\delta>0$ and $u_{0} \in \mathrm{L}_{\text {loc }}^{1}\left(\mathbb{R}^{N}\right) \cap \mathrm{L}^{\infty}(\{|x|>\delta\})$ such that

$$
u_{0}(x)=\mu|x|^{-\frac{2}{\alpha}} \text { a.e. on }\{|x|<\delta\},
$$

there exist $T>0$ and a solution $u \in \mathrm{L}_{\text {loc }}^{\alpha+1}\left((0, T) \times \mathbb{R}^{N}\right) \cap \mathrm{C}\left((0, T) \times\left(\mathbb{R}^{N} \backslash\{0\}\right)\right)$ of (1.1) in the sense of distributions, such that (1.12) holds and $u(0)=u_{0}$ in the sense (1.11). Moreover, $u(t)$ is singular at $x=0$ for all $t<T$ and satisfies (1.14).

There exist sign-changing self-similar solutions $U$ to which Theorem 1.4 applies, by Proposition 7.2. In this case, the solution $u$ is necessarily sign-changing for small time, see the discussion below. Moreover, Proposition 7.2 states that (1.15) can be achieved by a sign-changing profile for a sequence $\mu=\mu_{n} \rightarrow \infty$.

Note that, for a given solution $U$, Theorems 1.3 and 1.4 produce many different solutions of (1.1) with the same singularity. Indeed, we can choose $u_{0}$ arbitrarily for $|x|>\delta$ as long as $u_{0} \in \mathrm{L}^{\infty}(\{|x|>\delta\})$. That two different choices of $u_{0}$ produce two different solutions of (1.1) follows from (1.11).

We observe that using Remark 1.2 and Theorems 1.3 and 1.4 we do indeed obtain sign-changing solutions of (1.1) with persistent singularities, which are neither stationary nor self-similar. We may assume that $u_{0}$ is 
neither homogeneous, nor a stationary solution of (1.1). That $u$ is sign-changing is of course true by (1.11) if $u_{0}$ is sign-changing, and this is always possible since $u_{0}$ is prescribed only for $|x|<\delta$. Furthermore, in Theorem 1.3, if $U$ is sign-changing and $\delta$ is chosen sufficiently large, then $u_{0}$ is necessarily sign-changing. Finally, even if $u_{0}>0$, Theorem 1.4 produces sign-changing solutions. Indeed, suppose the profile $f$ is sign-changing (there exist such profiles, see [5], Thm. 1.3), and let $\tau, \varepsilon>0$ be such that $f(\tau)=-\varepsilon$. Given any $x_{0} \in \mathbb{R}^{N}$ such that $\left|x_{0}\right|=1$, it follows from (1.12) with $x=\tau \sqrt{t} x_{0}$ that

$$
\left|t^{\frac{1}{\alpha}} u\left(t, \tau \sqrt{t} x_{0}\right)+\varepsilon\right|=t^{\frac{1}{\alpha}}\left|u\left(t, \tau \sqrt{t} x_{0}\right)-U\left(t, \tau \sqrt{t} x_{0}\right)\right| \leq C\left(t^{\frac{1}{\alpha}}+\tau^{-\eta} t^{\frac{1}{\alpha}-\frac{\eta}{2}}\right) \leq \frac{\varepsilon}{2}
$$

for $t>0$ small, so that $u\left(t, \tau \sqrt{t} x_{0}\right) \leq-\frac{\varepsilon}{2} t^{-\frac{1}{\alpha}}<0$ for $t>0$ small.

We formalize some of the previous observations with the following corollary to Theorem 1.4.

Corollary 1.5. There exists a sequence $\left(\mu_{n}\right)_{n \geq 1} \subset(0, \infty), \mu_{n} \rightarrow \infty$, such that if $u_{0} \in \mathrm{L}_{\text {loc }}^{1}\left(\mathbb{R}^{N}\right) \cap \mathrm{L}^{\infty}(\{|x|>1\})$ satisfies

$$
u_{0}(x)=\mu_{n}|x|^{-\frac{2}{\alpha}} \text { a.e. on }\{|x|<\delta\},
$$

for some $\delta>0$ and $n \geq 1$, then there exist $T>0$ and a sign-changing solution $u \in \mathrm{L}_{\mathrm{loc}}^{\alpha+1}\left((0, T) \times \mathbb{R}^{N}\right) \cap$ $\mathrm{C}\left((0, T) \times\left(\mathbb{R}^{N} \backslash\{0\}\right)\right)$ of (1.1) in the sense of distributions, which is not self-similar (or stationary), such that $u(0)=u_{0}$ in the sense (1.11) and $u$ satisfies (1.14) for all $0<t<T$. In particular, $u(t)$ is singular at $x=0$ for all $0<t<T$.

In Theorem 1.4, the solution $u(t)$ has the spatial singularity $\mu|x|^{-\frac{2}{\alpha}}$ when $t=0$, and the singularity $\beta^{\frac{1}{\alpha}}|x|^{-\frac{2}{\alpha}}$ when $t>0$. Since $\mu$ can be chosen arbitrarily large by Proposition 7.2, we see that the singularity of $u$ at $t=0$ can be greater than the singularity at $t>0$.

Let $\alpha>0$ and let $u_{0} \in \mathrm{L}_{\text {loc }}^{\infty}\left(\mathbb{R}^{N} \backslash\{0\}\right) \cap \mathrm{L}^{\infty}(\{|x|>1\})$ equal $\mu|x|^{-\frac{2}{\alpha}}$ near the origin with $\mu>0$. If $\mu$ is sufficiently large, then there is no positive (possibly singular) solution of (1.1) with the initial value $u_{0}$. See ([5], Prop. A.1) or ([6], Cor. 2.7). (Note that this is not in contradiction with the results in [17-20], since all the positive, singular solutions of (1.1) constructed there have an initial value $u_{0}=u(0)$ which behaves like $\beta^{\frac{1}{\alpha}}|x|^{-\frac{2}{\alpha}}$ near the origin.) On the other hand, if $\alpha<\frac{4}{N-2}$, then there exist sign-changing, local in time solutions (regular for positive time) of (1.1) with the initial value $u_{0}$. See ([6], Thm. 5.1). It follows from Corollary 1.5 that, at least for certain arbitrarily large $\mu$ and under assumption (1.2), there also exist local in time, sign-changing solutions of (1.1) with the initial value $u_{0}$, which are singular at the origin for positive time.

We observe that for positive data $\left(U \geq 0\right.$ and $\left.u_{0} \geq 0\right)$, Theorem 1.3 is weaker than ([20], Thm. 1.1). Indeed, in ([20], Thm. 1.1), the singularity of $u$ can move with time, and $u_{0}$ need not be equal to $U(0)$ in a neighborhood of the origin, but sufficiently close to $U(0)$. A technical reason for this difference is that Theorem 1.3 allows sign-changing solutions so that we cannot apply the powerful comparison arguments used in [20].

Also, it is natural to ask if $u(t, x) \rightarrow 0$ as $|x| \rightarrow \infty$ in Theorems 1.3 and 1.4 , assuming $u_{0}(x) \rightarrow 0$ as $|x| \rightarrow \infty$. Our construction of the solution $u$ does not answer this question. The analogous property for perturbations of self-similar solutions with regular profile is true, see ([6], Thm. 5.1).

We now describe our strategy to prove Theorem 1.1. We construct $u$ as a perturbation of $U$ in the form

$$
u=U+w .
$$

The resulting equation for $w$ is

$$
\partial_{t} w-\Delta w=|U+w|^{\alpha}(U+w)-|U|^{\alpha} U .
$$

The leading term on the right-hand side of (1.17) is $(\alpha+1)|U|^{\alpha} w$, which by (1.7) behaves like $\beta(\alpha+1)|x|^{-2} w$ near the origin. This makes it delicate to apply a standard perturbation argument to (1.17). It turns out to 
be helpful to subtract the term $\beta(\alpha+1)|x|^{-2} w$ from both sides of the equation, leading to the following heat equation with inverse square potential

$$
\partial_{t} w-\Delta w-\beta(\alpha+1)|x|^{-2} w=\mathcal{M} w
$$

where

$$
\mathcal{M} w=|U+w|^{\alpha}(U+w)-|U|^{\alpha} U-\beta(\alpha+1)|x|^{-2} w .
$$

We observe that the operator $-\Delta-\beta(\alpha+1)|x|^{-2}$ in (1.18) has good properties only if

$$
\beta(\alpha+1)<\frac{(N-2)^{2}}{4},
$$

the constant in Hardy's inequality. Inequality (1.20) is equivalent to $\alpha<\alpha_{0}$, where $\alpha_{0}$ is given by (1.3). (See Lem. 2.1 below.) Note that $\alpha_{0}>\frac{2}{N-2}$, since $N>2$. Under the assumption (1.20), the operator $H$ on $L^{2}\left(\mathbb{R}^{N}\right)$ defined by

$$
\left\{\begin{array}{l}
D(H)=\left\{u \in \mathrm{H}^{1}\left(\mathbb{R}^{N}\right) ; \Delta u+\beta(\alpha+1)|x|^{-2} u \in \mathrm{L}^{2}\left(\mathbb{R}^{N}\right)\right\} \\
H u=\Delta u+\beta(\alpha+1)|x|^{-2} u, \quad u \in D(H)
\end{array}\right.
$$

is a negative self-adjoint operator, hence the generator of a $C_{0}$ semigroup of contractions $\left(e^{t H}\right)_{t \geq 0}$, which is an analytic semigroup on $\mathrm{L}^{2}\left(\mathbb{R}^{N}\right)$. Moreover, there exist two constants $A>0$ and $a>0$ such that the corresponding heat kernel $\mathcal{K}(t, x, y)$ satisfies the estimate

$$
0<\mathcal{K}(t, x, y) \leq A t^{-\frac{N}{2}} e^{-\frac{|x-y|^{2}}{a t}} h(t, x) h(t, y)
$$

where

$$
h(t, x)=\left(1+\frac{\sqrt{t}}{|x|}\right)^{\eta}
$$

and $\eta$ is given by (1.13). See ([10], Thm. 1.2), ([12], Thm. 3), ([13], Thm. 3.10).

Using the kernel $\mathcal{K}$ we write equation $(1.18)$ in the integral form

$$
w(t)=\int_{\mathbb{R}^{N}} \mathcal{K}(t, x, y) w_{0}(y) \mathrm{d} y+\int_{0}^{t} \int_{\mathbb{R}^{N}} \mathcal{K}(t-s, x, y) \mathcal{M} w(s, y) \mathrm{d} y \mathrm{~d} s
$$

where $w_{0}=u(0)-U(0)$. Since $\mathcal{K}$ is bounded from below by a term similar to the right-hand side of (1.22), it follows that $\mathcal{K}(t, x, y)$ has the singularity $|x|^{-\eta}$ as $|x| \rightarrow 0$ and similarly in $y$. Thus we see that the kernel of the operator $e^{t H}$ associated with equation (1.18) is more singular than the heat kernel associated with equation (1.17). Of course, the right-hand side of (1.18) is less singular than the right-hand side of (1.17). In fact the worst term in $\mathcal{M} w$ is of order $\left(\frac{|x|}{|x|+\sqrt{t}}\right)^{\omega}|x|^{-2} w$, where $\omega>0$ is given by (3.14) below. (See (4.14), (4.16), (4.17).) At positive times, this term is better than $|x|^{-\frac{2}{\alpha}} w$. However, as $t \rightarrow 0$, it behaves like $|x|^{-\frac{2}{\alpha}} w$. This, combined with the singularity of the kernel, excludes the possibility of carrying out a standard contraction mapping argument based on (1.24). Our solution to this difficulty is taken from [6] and involves a contraction mapping argument in a class of functions $w$ that are sufficiently small as $(t, x) \rightarrow(0,0)$ so as to balance the singularity of $\mathcal{M} w$. The key point is to find such a class which is preserved by the iterative process. The fixed 
point $w$ thus obtained satisfies the integral equation (1.24) and in fact solves (1.18) in the sense of distributions. Therefore $u=U+w$ satisfies (1.10) in the sense of distributions.

We do not know if the condition $\alpha<\alpha_{0}$ in Theorem 1.1 is necessary. However, if $\alpha>\alpha_{0}($ i.e. $\beta(\alpha+1)>$ $\left.\frac{(N-2)^{2}}{4}\right)$, then our proof breaks down from the beginning, since in this case the linear heat equation with potential $\beta(\alpha+1)|x|^{-2}$ is ill-posed, see [1,23].

The results in this paper are motivated by our article [6] where we prove an analogue of Theorem 1.4 where the self-similar solution $U$ has a regular profile. Such self-similar solutions have a singularity at $(t, x)=(0,0)$, which introduces some limitations in our results. In particular, we are led to consider in [6] initial values $w_{0}$ that equal $U(0, \cdot)$ in a neighborhood of the origin. The same limitation appears here, and we do not know if it is technical or not.

The rest of this paper is organized as follows. Section 2 is devoted to some properties of the parameters we use throughout the paper. In Section 3, we establish some specific estimates for the nonhomogeneous heat equation with inverse square potential. In Section 4, we introduce the setting for the fixed point argument that we use in Section 5 to prove Theorem 1.1. In Section 6, we give a description of the radially symmetric, stationary solutions of (1.1), showing the existence of sign-changing solutions. In Section 7, we deduce Theorems 1.3 and 1.4 from Theorem 1.1. We collect in Appendix A some general properties, concerning mostly the linear heat equation with inverse square potential, that we use in the paper and for which we did not find a reference.

\section{Elementary inEQUALITIES}

This section is devoted to the following elementary properties.

Lemma 2.1. Suppose $N \geq 3$, and let $\alpha_{0}>0$ be defined by (1.3) and $\Lambda \in \mathbb{R}$ by

$$
\Lambda=\left(\frac{1}{\alpha}-\frac{N-2}{4}\right)^{2}-\left(\frac{N-2}{2}-\frac{1}{\alpha}\right) .
$$

The following properties hold.

(i) $\alpha_{0}$ satisfies

$$
\frac{2}{N-2}<\alpha_{0}<\frac{4}{N-2}
$$

(ii) For $0<\alpha<\frac{4}{N-2}$, the three properties

$$
\begin{gathered}
\alpha<\alpha_{0}, \\
\Lambda>0,
\end{gathered}
$$

and (1.20) (where $\beta$ is given by (1.4)), are equivalent.

(iii) Let $0<\alpha<\alpha_{0}$ and let $\Lambda>0$ be given by (2.1). If $\mu_{1}, \mu_{2}$ are defined by

$$
\begin{aligned}
& \mu_{1}=\frac{1}{\alpha}-\frac{N-2}{4}-\sqrt{\Lambda} \\
& \mu_{2}=\frac{1}{\alpha}-\frac{N-2}{4}+\sqrt{\Lambda}
\end{aligned}
$$

then $0<\mu_{1}<\mu_{2}$. Moreover, $\frac{(N-2)^{2}}{4}-\beta(\alpha+1)>0$ by Property (ii) above, and if $\rho$ is given by (1.8), then

$$
\rho=2 \mu_{1}
$$


so that $\rho>0$.

Proof. Since

$$
2 \sqrt{N-1}=\sqrt{N^{2}-(N-2)^{2}}
$$

we see that $2 \sqrt{N-1}<N$. Therefore $N-4+2 \sqrt{N-1}<2 N-4$, hence $\alpha_{0}>\frac{2}{N-2}$. Moreover,

$$
2 \sqrt{N-1}>2,
$$

so that $N-4+2 \sqrt{N-1}>N-2$, hence $\alpha_{0}<\frac{4}{N-2}$. This proves Property (2.2).

We now prove Property (ii). We have

$$
\frac{(N-2)^{2}}{4}-\beta(\alpha+1)=4 \Lambda
$$

so that (2.4) and (1.20) are equivalent. We write

$$
\frac{1}{\alpha_{0}}=\frac{N-2}{4}+\frac{\sqrt{N-1}-1}{2}
$$

and

$$
\frac{1}{\alpha}=\frac{1}{\alpha_{0}}+\varepsilon
$$

so that

$$
\Lambda=\varepsilon^{2}+\varepsilon \sqrt{N-1}
$$

It follows that $\Lambda>0$ if and only if either $\varepsilon>0$, i.e. $\alpha<\alpha_{0}$, or else $\varepsilon<-\sqrt{N-1}$. In this last case,

$$
\frac{1}{\alpha}<\frac{1}{\alpha_{0}}-\sqrt{N-1}=\frac{N-2}{4}-\frac{1}{2}-\frac{\sqrt{N-1}}{2}<\frac{N-2}{4}
$$

so that $\alpha>\frac{4}{N-2}$. Thus we see that (2.3) and (2.4) are equivalent.

To prove Property (iii), we observe that $\mu_{2}>\mu_{1}$. Moreover, $\Lambda<\left(\frac{1}{\alpha}-\frac{N-2}{4}\right)^{2}$. Thus $\sqrt{\Lambda}<\frac{1}{\alpha}-\frac{N-2}{4}$ so that $\mu_{1}>0$. Finally, formula (2.8) yields $\rho=2 \mu_{1}$.

\section{The nonhomogeneous heat EQUATION WITh InVERSE SQUARE POTENTIAL}

In this section, we assume (1.2), and we use the operator $H$ defined by (1.21) and the corresponding semigroup $\left(e^{t H}\right)_{t \geq 0}$ with the kernel $\mathcal{K}(t, x, y)$. We let $\rho, \eta>0$ be defined by (1.8) and (1.13), respectively. This section is devoted to estimates of

$$
\int_{0}^{t} e^{(t-s) H} f(s) \mathrm{d} s
$$

for some specific right-hand sides $f$. 
Lemma 3.1. Let $c \geq 1,0 \leq b \leq 2$ and $\kappa \geq 0(\kappa>0$ if $b=2)$ satisfy

$$
b+(c-1) \eta-\kappa<2 .
$$

Since $2 \eta<N-2$ by (1.13), we have $b+(c+1) \eta-\kappa<N$, and we fix

$$
0 \leq \varepsilon<\min \left\{\frac{1}{4}, N-(b+(1+c) \eta-\kappa)\right\} .
$$

Define $\Psi(t)$ for $t>0$ by

$$
\Psi(t, x)=h(t, x)^{c}|x|^{-b}\left(\frac{|x|}{|x|+\sqrt{t}}\right)^{\kappa}, \quad x \in \mathbb{R}^{N}
$$

where $h$ is given by (1.23). It follows that for every $m>\kappa-2$ there exists $B_{m}>0$ such that for all $0<r \leq \infty$,

$$
\int_{0}^{t} e^{(t-s) H} 1_{\{|y|<r\}} \Psi(s, \cdot) s^{\frac{m}{2}} \mathrm{~d} s \leq B_{m} r^{\varepsilon} t^{\frac{m+2-b}{2}}|x|^{-\varepsilon} h(t, x),
$$

for all $t>0$ and $x \in \mathbb{R}^{N}$, with $B_{m} \rightarrow 0$ as $m \rightarrow \infty$.

Proof. We write using (1.22),

$$
\begin{aligned}
e^{(t-s) H} 1_{\{|y|<r\}} \Psi(s)(x) & \leq A h(t-s, x)(t-s)^{-\frac{N}{2}} \int_{\mathbb{R}^{N}} e^{-\frac{|x-y|^{2}}{a(t-s)}} 1_{\{|y|<r\}} h(t-s, y) \Psi(s, y) \mathrm{d} y \\
& =A(a \pi)^{\frac{N}{2}} h(t-s, x) e^{\frac{a}{4}(t-s) \Delta}\left[1_{\{|y|<r\}} h(t-s, \cdot) \Psi(s, \cdot)\right](x),
\end{aligned}
$$

so that

$$
e^{(t-s) H} 1_{\{|y|<r\}} \Psi(s) \leq C h(t-s, x) e^{\frac{a}{4}(t-s) \Delta}\left[1_{\{|y|<r\}} h(t-s, \cdot) h(s, \cdot)^{c}|\cdot|^{-b}\left(\frac{|\cdot|}{|\cdot|+\sqrt{s}}\right)^{\kappa}\right] .
$$

Since

$$
h(t-s, y) h(s, y)^{c} \leq C\left(1+\frac{(t-s)^{\frac{\eta}{2}}}{|y|^{\eta}}\right)\left(1+\frac{s^{\frac{\eta c}{2}}}{|y|^{\eta c}}\right) \leq C\left(1+\frac{(t-s)^{\frac{\eta}{2}}}{|y|^{\eta}}+\frac{s^{\frac{\eta c}{2}}}{|y|^{\eta c}}+\frac{(t-s)^{\frac{\eta}{2}} s^{\frac{\eta c}{2}}}{|y|^{(1+c) \eta}}\right),
$$

we deduce that

$$
e^{(t-s) H} 1_{\{|y|<r\}} \Psi(s) \leq C h(t-s, x)\left[I_{1}+(t-s)^{\frac{\eta}{2}} I_{2}+s^{\frac{\eta c}{2}} I_{3}+(t-s)^{\frac{\eta}{2}} s^{\frac{\eta c}{2}} I_{4}\right]
$$

where

$$
\begin{aligned}
& I_{1}=e^{\frac{a}{4}(t-s) \Delta}\left(1_{\{|y|<r\}}|\cdot|^{-b}\left(\frac{|\cdot|}{|\cdot|+\sqrt{s}}\right)^{\kappa}\right), \\
& I_{2}=e^{\frac{a}{4}(t-s) \Delta}\left(1_{\{|y|<r\}}|\cdot|^{-b-\eta}\left(\frac{|\cdot|}{|\cdot|+\sqrt{s}}\right)^{\kappa}\right), \\
& I_{3}=e^{\frac{a}{4}(t-s) \Delta}\left(1_{\{|y|<r\}}|\cdot|^{-b-\eta c}\left(\frac{|\cdot|}{|\cdot|+\sqrt{s}}\right)^{\kappa}\right), \\
& I_{4}=e^{\frac{a}{4}(t-s) \Delta}\left(1_{\{|y|<r\}}|\cdot|^{-b-(1+c) \eta}\left(\frac{|\cdot|}{|\cdot|+\sqrt{s}}\right)^{\kappa}\right) .
\end{aligned}
$$


We note that

$$
1_{\{|y|<r\}} \leq r^{\varepsilon}|y|^{-\varepsilon}
$$

and we recall that if $0 \leq p<N$, then

$$
e^{t \Delta}|\cdot|^{-p} \leq C\left(t+|x|^{2}\right)^{-\frac{p}{2}}
$$

See e.g. ([4], Cor. 8.3).

Let $\kappa_{1}=\min \{\kappa, b\}$. We have

$$
\begin{aligned}
I_{1} & \leq r^{\varepsilon} e^{\frac{a}{4}(t-s) \Delta}\left(|\cdot|^{-b-\varepsilon}\left(\frac{|\cdot|}{|\cdot|+\sqrt{s}}\right)^{\kappa_{1}}\right) \leq r^{\varepsilon} s^{-\frac{\kappa_{1}}{2}} e^{\frac{a}{4}(t-s) \Delta}\left(|\cdot|^{-\left(b+\varepsilon-\kappa_{1}\right)}\right) \\
& \leq C r^{\varepsilon} s^{-\frac{\kappa_{1}}{2}}\left(t-s+|x|^{2}\right)^{-\frac{b+\varepsilon-\kappa_{1}}{2}} \leq C r^{\varepsilon}|x|^{-\varepsilon} s^{-\frac{\kappa_{1}}{2}}(t-s)^{-\frac{b-\kappa_{1}}{2}},
\end{aligned}
$$

where we used (3.6), (3.7) and the property $0 \leq b+\varepsilon-\kappa_{1} \leq b+\varepsilon \leq \frac{9}{4}<N$.

Similarly, letting $\kappa_{2}=\min \{\kappa, b+\eta\}$,

$$
I_{2} \leq r^{\varepsilon} s^{-\frac{\kappa_{2}}{2}} e^{\frac{a}{4}(t-s) \Delta}\left(|\cdot|^{-b-\eta-\varepsilon+\kappa_{2}}\right) \leq C r^{\varepsilon} s^{-\frac{\kappa_{2}}{2}}\left(t-s+|x|^{2}\right)^{-\frac{b+\eta+\varepsilon-\kappa_{2}}{2}} \leq C r^{\varepsilon}|x|^{-\varepsilon} s^{-\frac{\kappa_{2}}{2}}(t-s)^{-\frac{b+\eta-\kappa_{2}}{2}},
$$

where we used (3.6), (3.7) and the property

$$
0 \leq b+\eta+\varepsilon-\kappa_{2} \leq b+\eta+\varepsilon \leq \frac{9}{4}+\eta \leq \frac{9}{4}+\frac{N-2}{2}<N .
$$

Next, setting $\kappa_{3}=\min \{\kappa, b+c \eta\}$,

$$
\begin{aligned}
I_{3} & \leq r^{\varepsilon} s^{-\frac{\kappa_{3}}{2}} e^{\frac{a}{4}(t-s) \Delta}\left(|\cdot|^{-b-c \eta-\varepsilon+\kappa_{3}}\right) \leq C r^{\varepsilon} s^{-\frac{\kappa_{3}}{2}}\left(t-s+|x|^{2}\right)^{-\frac{b+c \eta+\varepsilon-\kappa_{3}}{2}} \\
& \leq C r^{\varepsilon}|x|^{-\varepsilon} s^{-\frac{\kappa_{3}}{2}}(t-s)^{-\frac{b+(c-1) \eta-\kappa_{3}}{2}}\left(t-s+|x|^{2}\right)^{-\frac{\eta}{2}} \\
& \leq C r^{\varepsilon}|x|^{-\varepsilon} s^{-\frac{\kappa_{3}}{2}}(t-s)^{-\frac{b+(c-1) \eta-\kappa_{3}}{2}} \frac{1}{|x|^{\eta} h(t-s, x)} .
\end{aligned}
$$

Here we used (3.6), (3.7) and the property $0 \leq b+c \eta+\varepsilon-\kappa_{3}<N$. The last inequality is immediate if $\kappa_{3}=b+c \eta$; and if $\kappa_{3}=\kappa$, it follows from $b+c \eta+\varepsilon-\kappa \leq b+(1+c) \eta+\varepsilon-\kappa<N$ by (3.2).

Furthermore, setting $\kappa_{4}=\min \{\kappa, b+(1+c) \eta\}$,

$$
\begin{aligned}
I_{4} & \leq r^{\varepsilon} s^{-\frac{\kappa_{4}}{2}} e^{\frac{a}{4}(t-s) \Delta}\left(|\cdot|^{-b-(1+c) \eta-\varepsilon+\kappa_{4}}\right) \leq C r^{\varepsilon} s^{-\frac{\kappa_{4}}{2}}\left(t-s+|x|^{2}\right)^{-\frac{b+(1+c) \eta+\varepsilon-\kappa_{4}}{2}} \\
& \leq C r^{\varepsilon}|x|^{-\varepsilon} s^{-\frac{\kappa_{4}}{2}}(t-s)^{-\frac{b+c \eta-\kappa_{4}}{2}}\left(t-s+|x|^{2}\right)^{-\frac{\eta}{2}} \leq C r^{\varepsilon}|x|^{-\varepsilon} s^{-\frac{\kappa_{4}}{2}}(t-s)^{-\frac{b+c \eta-\kappa_{4}}{2}} \frac{1}{|x|^{\eta} h(t-s, x)} .
\end{aligned}
$$

In (3.11) we used (3.6), (3.7) and the property $0 \leq b+(1+c) \eta+\varepsilon-\kappa_{4}<N$. The last inequality is immediate if $\kappa_{4}=b+(1+c) \eta$; and if $\kappa_{4}=\kappa$, it follows from (3.2). We deduce from (3.5), (3.8), (3.9), (3.10) and (3.11) that

$$
\begin{aligned}
r^{-\varepsilon}|x|^{\varepsilon} e^{(t-s) H} 1_{\{|y|<r\}} \Psi(s) \leq & C h(t-s, x)\left[s^{-\frac{\kappa_{1}}{2}}(t-s)^{-\frac{b-\kappa_{1}}{2}}+s^{-\frac{\kappa_{2}}{2}}(t-s)^{-\frac{b-\kappa_{2}}{2}}\right] \\
& +C|x|^{-\eta}\left[s^{\frac{\eta n-\kappa_{3}}{2}}(t-s)^{-\frac{b+(c-1) \eta-\kappa_{3}}{2}}+s^{\frac{\eta c-\kappa_{4}}{2}}(t-s)^{-\frac{b+(c-1) \eta-\kappa_{4}}{2}}\right] .
\end{aligned}
$$


Since $h(t-s, x) \leq h(t, x)$ and $|x|^{-\eta} \leq t^{-\frac{\eta}{2}} h(t, x)$, we deduce that

$$
\begin{aligned}
r^{-\varepsilon}|x|^{\varepsilon} e^{(t-s) H} 1_{\{|y|<r\}} \Psi(s) \leq & C h(t, x)\left[s^{-\frac{\kappa_{1}}{2}}(t-s)^{-\frac{b-\kappa_{1}}{2}}+s^{-\frac{\kappa_{2}}{2}}(t-s)^{-\frac{b-\kappa_{2}}{2}}\right. \\
& \left.+t^{-\frac{\eta}{2}}\left(s^{\frac{\eta c-\kappa_{3}}{2}}(t-s)^{-\frac{b+(c-1) \eta-\kappa_{3}}{2}}+s^{\frac{\eta c-\kappa_{4}}{2}}(t-s)^{-\frac{b+(c-1) \eta-\kappa_{4}}{2}}\right)\right] .
\end{aligned}
$$

Multiplying by $s^{\frac{m}{2}}$ and integrating in $s \in(0, t)$, we obtain (3.4) with

$$
B_{m}=C \sum_{j=1}^{2} \int_{0}^{1} \sigma^{\frac{m-\kappa_{j}}{2}}(1-\sigma)^{-\frac{b-\kappa_{j}}{2}} \mathrm{~d} \sigma+C \sum_{j=3}^{4} \int_{0}^{1} \sigma^{\frac{m+\eta c-\kappa_{j}}{2}}(1-\sigma)^{-\frac{b+(c-1) \eta-\kappa_{j}}{2}} \mathrm{~d} \sigma .
$$

The integrals in (3.12) are finite. Indeed, for all $j \in\{1,2,3,4\}$,

$$
\min \left\{\frac{m-\kappa_{j}}{2}, \frac{m+\eta c-\kappa_{j}}{2}\right\} \geq \frac{m-\kappa}{2}>-1 .
$$

Moreover, $\frac{b-\kappa_{j}}{2}<\frac{b}{2} \leq 1$ for $j=1,2$. Furthermore, if $\kappa_{j}=\kappa$ for $j=3$ or $j=4$, then

$$
\frac{b+(c-1) \eta-\kappa_{j}}{2}=\frac{b+(c-1) \eta-\kappa}{2}<1
$$

by (3.1). If $\kappa_{3}=b+c \eta$, then $\frac{b+(c-1) \eta-\kappa_{3}}{2}=-\frac{\eta}{2}<0<1$. Similarly if $\kappa_{4}=b+(1+c) \eta$, then $\frac{b+(c-1) \eta-\kappa_{4}}{2}=$ $-\eta<0<1$. Finally, the property $B_{m} \rightarrow 0$ follows by dominated convergence.

Lemma 3.2. Let

$$
\begin{aligned}
& \left(b_{1}, c_{1}, \kappa_{1}\right)=(2,1, \rho), \\
& \left(b_{2}, c_{2}, \kappa_{2}\right)=(0,1+\alpha, 0), \\
& \left(b_{3}, c_{3}, \kappa_{3}\right)=\left(\frac{2(\alpha-1)}{\alpha}, 2,0\right), \\
& \left(b_{4}, c_{4}, \kappa_{4}\right)=(2,1, \alpha \rho) .
\end{aligned}
$$

It follows that $b_{3}<2$ and that the triplets $\left(b_{j}, c_{j}, \kappa_{j}\right)$ satisfy $(3.1)$ for $j=1,2,3,4$.

Proof. We first note that by (1.13)

$$
\eta<\frac{N-2}{2}<\frac{2}{\alpha}
$$

since $\alpha<\frac{4}{N-2}$. Next,

$$
b_{1}+\left(c_{1}-1\right) \eta-\kappa_{1}=2-\rho<2 .
$$

For $j=2$,

$$
b_{2}+\left(c_{2}-1\right) \eta-\kappa_{2}=\alpha \eta<2
$$

by (3.13). For $j=3$,

$$
b_{3}+\left(c_{3}-1\right) \eta-\kappa_{3}=2-\frac{2}{\alpha}+\eta<2
$$


by (3.13). For $j=4$,

$$
b_{4}+\left(c_{4}-1\right) \eta-\kappa_{4}=2-\alpha \rho<2 .
$$

This completes the proof.

Lemma 3.3. Let

$$
\omega=\rho \min \{1, \alpha\}
$$

and let

$$
g(t, x)=\left(\frac{|x|}{|x|+\sqrt{t}}\right)^{\omega}|x|^{-2}+(\boldsymbol{M} h(t, x))^{\alpha}+\boldsymbol{M} \widetilde{g}(t, x)
$$

for a.a. $t>0, x \in \mathbb{R}^{N}$, where $M \geq 0, h$ is given by (1.23), and

$$
\widetilde{g}(t, x)= \begin{cases}0 & \alpha \leq 1 \\ |x|^{-\frac{2(\alpha-1)}{\alpha}} h(t, x) & \alpha>1 .\end{cases}
$$

It follows that there exists a constant $\boldsymbol{A}$ such that

$$
\begin{aligned}
g(t, x) & \leq \boldsymbol{A}\left(1+|x|^{-2}\right) \\
g(t, x) h(s, x) & \leq \boldsymbol{A}\left(1+|x|^{-\frac{N+2}{2}}\right) \\
g(t, x) h(s, x) h(\sigma, x) & \leq \boldsymbol{A}\left(1+|x|^{-N+\vartheta}\right)
\end{aligned}
$$

for a.a. $t, s, \sigma \in(0,1)$ and $x \in \mathbb{R}^{N}$, where

$$
\vartheta=\frac{N-2}{2}-\eta>0
$$

Moreover, there exists $\varepsilon_{0}>0$ such that if $0 \leq \varepsilon<\varepsilon_{0}$ is fixed, then for every $m>\max \{\rho, \alpha \rho\}-2$,

$$
\int_{0}^{t} e^{(t-s) H} 1_{\{|y|<r\}} g(s) h(s) s^{\frac{m}{2}} \mathrm{~d} s \leq R_{m} r^{\varepsilon} t^{\frac{m}{2}}|x|^{-\varepsilon} h(t, x)
$$

where

$$
R_{m} \underset{m \rightarrow \infty}{\longrightarrow} 0
$$

Proof. We first prove (3.16)-(3.18). It follows from (1.23) and (3.15) that for $0 \leq t \leq 1$

$$
\begin{aligned}
g(t, x) & \leq C\left(1+|x|^{-2}+|x|^{-\alpha \eta}+|x|^{-2+\frac{2}{\alpha}-\eta}\right) \\
g(t, x) h(s, x) & \leq C\left(1+|x|^{-2-\eta}+|x|^{-(\alpha+1) \eta}+|x|^{-2+\frac{2}{\alpha}-2 \eta}\right) \\
g(t, x) h(s, x) h(\sigma, x) & \leq C\left(1+|x|^{-2-2 \eta}+|x|^{-(\alpha+2) \eta}+|x|^{-2+\frac{2}{\alpha}-3 \eta}\right)
\end{aligned}
$$


Since

$$
\alpha<\frac{4}{N-2} \text { and } \quad \eta=\frac{N-2}{2}-\vartheta<\frac{N-2}{2},
$$

we see that $\eta \alpha<2$ and $\frac{2}{\alpha}-\eta>0$, so that (3.16) follows from (3.22). Using again (3.25), we obtain $2+\eta<\frac{N+2}{2}$, $(\alpha+1) \eta<\frac{N+2}{2}$ and $2-\frac{2}{\alpha}+2 \eta<\frac{N+2}{2}$, hence (3.17) follows from (3.23). Moreover, (3.25) yields

$$
\begin{gathered}
2+2 \eta=N-2 \vartheta \leq N-\vartheta, \\
(\alpha+2) \eta \leq N-\vartheta \frac{2 N}{N-2} \leq N-\vartheta, \\
2-\frac{2}{\alpha}+3 \eta \leq N-3 \vartheta \leq N-\vartheta,
\end{gathered}
$$

so that (3.18) follows from (3.24).

Estimate (3.20) follows from Lemma 3.1. For the term $\left(\frac{|x|}{|x|+\sqrt{t}}\right)^{\omega}|x|^{-2}$ we apply Lemma 3.1 with $(b, c, \kappa)=$ $(2,1, \rho)$ if $\omega=\rho$ and $(b, c, \kappa)=(2,1, \alpha \rho)$ if $\omega=\alpha \rho$. This is possible by Lemma 3.2. For the term $h^{\alpha}$ we apply Lemma 3.1 with $(b, c, \kappa)=(0,1+\alpha, 0)$. This is again possible by Lemma 3.2. Finally for the term $\widetilde{g}$, we need only consider the case $\alpha>1$ and we apply Lemma 3.1 with $(b, c, \kappa)=\left(\frac{2(\alpha-1)}{\alpha}, 2,0\right)$, which is possible by Lemma 3.2.

\section{THE SETTING FOR THE FIXED-POINT ARGUMENT}

In this section, we assume (1.2), and we use the operator $H$ defined by (1.21) and the corresponding semigroup $\left(e^{t H}\right)_{t \geq 0}$ with the kernel $\mathcal{K}(t, x, y)$. We introduce the framework for the fixed-point argument that we use for the proof of Theorem 1.1.

We begin with the definition of several auxiliary functions. Let $\delta>0$, and set

$$
a_{j}=2^{-j} \delta
$$

for $j \geq 0$. Define the sequence $\left(\chi_{j}\right)_{j \geq 0} \subset \mathrm{L}^{\infty}\left(\mathbb{R}^{N}\right)$ by

$$
\chi_{j}(x)= \begin{cases}0 & |x| \leq a_{j} \\ 1 & |x|>a_{j} .\end{cases}
$$

Given $T>0$ and an integer $m \geq 1$, we set

$$
\Theta(t, x)=h(t, x)\left(t^{\frac{m}{2}}+\sum_{j=1}^{m} t^{\frac{j-1}{2}} \chi_{j}\right)
$$

for $0 \leq t \leq T$ and $x \in \mathbb{R}^{N}$, where $h$ is given by (1.23). Given $\boldsymbol{M}>0$, we define

$$
\mathcal{E}=\left\{w \in \mathrm{L}_{\mathrm{loc}}^{1}\left((0, T) \times \mathbb{R}^{N}\right) ;|w| \leq \boldsymbol{M \Theta}\right\},
$$

and

$$
\mathrm{d}(w, z)=\left\|\frac{w-z}{\Theta}\right\|_{L^{\infty}\left((0, T) \times \mathbb{R}^{N}\right)}, \quad w, z \in \mathcal{E},
$$


so that $(\mathcal{E}, \mathrm{d})$ is a complete metric space.

Lemma 4.1. Let $\delta>0$. With the notation (4.1)-(4.2), it follows that there exists $\boldsymbol{B}_{1}>0$ such that for all $j \geq 0$ and all $0 \leq t, s \leq 1$

$$
e^{t H} \chi_{j} \leq \boldsymbol{B}_{1} h(t, x)\left(e^{-\frac{a_{j+1}^{2}}{2 a t}}+\chi_{j+1}\right)
$$

where $a$ is the constant in (1.22) and $h$ is given by (1.23).

Proof. Applying (1.22),

$$
\int_{\mathbb{R}^{N}} \mathcal{K}(t, x, y) \chi_{j}(y) \mathrm{d} y \leq C t^{-\frac{N}{2}} h(t, x) \int_{\left\{|y|>a_{j}\right\}} e^{-\frac{|x-y|^{2}}{a t}} h(t, y) \mathrm{d} y .
$$

We also write

$$
\int_{\mathbb{R}^{N}} \mathcal{K}(t, x, y) \chi_{j}(y) \mathrm{d} y \leq C t^{-\frac{N}{2}} h(t, x) \int_{\mathbb{R}^{N}} e^{-\frac{|y|^{2}}{a t}} h(t, x-y) \mathrm{d} y .
$$

We deduce from (4.8) that

$$
\begin{aligned}
\int_{\mathbb{R}^{N}} \mathcal{K}(t, x, y) \chi_{j}(y) \mathrm{d} y & \leq C t^{-\frac{N}{2}} h(t, x) \int_{\mathbb{R}^{N}} e^{-\frac{|y|^{2}}{a t}}\left(1+\frac{\sqrt{t}}{|x-y|}\right)^{\eta} \mathrm{d} y \\
& =C h(t, x) \int_{\mathbb{R}^{N}} e^{-\frac{|y|^{2}}{a}}\left(1+\frac{1}{|(x / \sqrt{t})-y|}\right)^{\eta} \mathrm{d} y .
\end{aligned}
$$

Since $\eta<N$ by (1.13), we see that

$$
\int_{\mathbb{R}^{N}} e^{-\frac{|y|^{2}}{a}}\left(1+\frac{1}{|z-y|}\right)^{\eta} \leq 2^{\eta} \int_{\mathbb{R}^{N}} e^{-\frac{|y|^{2}}{a}}+2^{\eta} \int_{|z-y|<1}|z-y|^{-\eta} \leq C
$$

independent of $z \in \mathbb{R}^{N}$, and it follows that

$$
\int_{\mathbb{R}^{N}} \mathcal{K}(t, x, y) \chi_{j}(y) \mathrm{d} y \leq C h(t, x)
$$

for all $x \in \mathbb{R}^{N}$. In particular, if $|x|>a_{j+1}$, then

$$
\int_{\mathbb{R}^{N}} \mathcal{K}(t, x, y) \chi_{j}(y) \mathrm{d} y \leq C h(t, x) \chi_{j+1}(x) .
$$

Next, if $|x| \leq a_{j+1}$ and $|y| \geq a_{j}$, then

$$
|x-y| \geq|y|-a_{j+1}=|y|-\frac{1}{2} a_{j} \geq \frac{1}{2}|y| \geq a_{j+1}
$$

and so

$$
e^{-\frac{|x-y|^{2}}{a t}}=e^{-\frac{|x-y|^{2}}{2 a t}} e^{-\frac{|x-y|^{2}}{2 a t}} \leq e^{-\frac{|y|^{2}}{8 a t}} e^{-\frac{a_{j+1}^{2}}{2 a t}} .
$$


If $|x| \leq a_{j+1}$, then we deduce from (4.7), (4.10), and $h(t, \cdot) \leq h(1, \cdot)$ that

$$
\int_{\mathbb{R}^{N}} \mathcal{K}(t, x, y) \chi_{j}(y) \mathrm{d} y \leq C h(t, x) e^{-\frac{a_{j+1}^{2}}{2 a t}} t^{-\frac{N}{2}} \int_{\mathbb{R}^{N}} e^{-\frac{|y|^{2}}{8 a t}} h(1, y) \mathrm{d} y \leq C h(t, x) e^{-\frac{a_{j+1}^{2}}{2 a t}}
$$

Then (4.6) follows from (4.9) and (4.11).

Lemma 4.2. Let $S>0$ and let $U \in \mathrm{L}^{1}\left((0, S) \times \mathbb{R}^{N}\right)+\mathrm{L}^{\infty}\left((0, S) \times \mathbb{R}^{N}\right)$ satisfy (1.7) for a.a. $0<t<S$ and $x \neq 0$, where $\rho$ is given by (1.8). Let $T \leq S$,

$$
0<T<\frac{1}{4}
$$

$\delta>0, \boldsymbol{M}>0$, and let $\mathcal{E}$ be defined by (4.4). It follows that $\mathcal{M} w \in \mathrm{L}_{\mathrm{loc}}^{1}\left((0, T) \times \mathbb{R}^{N}\right)$ for all $w \in \mathcal{E}$, where $\mathcal{M} w$ is defined by (1.19). Moreover,

$$
|\mathcal{M} w(t, \cdot)-\mathcal{M} z(t, \cdot)| \leq \boldsymbol{B}_{2}|w(t, \cdot)-z(t, \cdot)| g(t, \cdot)
$$

for all $w, z \in \mathcal{E}$, where $g(t, x)$ is given by (3.15) and $\boldsymbol{B}_{2}$ is independent of $T, m, \boldsymbol{M}, w$ and $z$.

Proof. Let $w, z \in \mathcal{E}$. Set

$$
f(s)=|s|^{\alpha} s
$$

and define

$$
Z(x)=\beta^{\frac{1}{\alpha}}|x|^{-\frac{2}{\alpha}}, \quad V=U-Z .
$$

It follows that

$$
\mathcal{M} w=f(Z+V+w)-f(Z+V)-f^{\prime}(Z) w,
$$

so that

$$
\begin{aligned}
\mathcal{M} w-\mathcal{M} z & =f(Z+V+w)-f(Z+V+z)-f^{\prime}(Z)(w-z) \\
& =(w-z) \int_{0}^{1}\left[f^{\prime}(Z+V+s w+(1-s) z)-f^{\prime}(Z)\right] \mathrm{d} s .
\end{aligned}
$$

Since $f^{\prime}(s)=(\alpha+1)|s|^{\alpha}$ and

$$
\left.|| x\right|^{\alpha}-|y|^{\alpha} \mid \leq \begin{cases}\alpha\left(|x|^{\alpha-1}+|y|^{\alpha-1}\right)|x-y| & \text { if } \alpha \geq 1 \\ |x-y|^{\alpha} & \text { if } 0<\alpha \leq 1\end{cases}
$$

for all $x, y \in \mathbb{R}$, we deduce from (4.15) that

$$
|\mathcal{M} w-\mathcal{M} z| \leq C|w-z| \times \begin{cases}|V|^{\alpha}+|w|^{\alpha}+|z|^{\alpha}+Z^{\alpha-1}(|V|+|w|+|z|) & \text { if } \alpha \geq 1 \\ |V|^{\alpha}+|w|^{\alpha}+|z|^{\alpha} & \text { if } 0<\alpha \leq 1\end{cases}
$$


By (1.7)

$$
|V(t, x)| \leq C\left[\left(\frac{|x|}{|x|+\sqrt{t}}\right)^{\rho}|x|^{-\frac{2}{\alpha}}+1\right] .
$$

On the other hand, (4.3) and (4.12) yield

$$
|w|+|z| \leq 2 \boldsymbol{M} \Theta(t, x) \leq 2 \boldsymbol{M} h(t, x) \sum_{j=0}^{m} t^{\frac{j}{2}}<4 \boldsymbol{M} h(t, x) .
$$

if $t<T$. From (4.17) and (4.18) we get (recall that $h \geq 1$ )

$$
|V|^{\alpha}+|w|^{\alpha}+|z|^{\alpha} \leq C\left(\left(\frac{|x|}{|x|+\sqrt{t}}\right)^{\alpha \rho}|x|^{-2}+(\boldsymbol{M} h(t, x))^{\alpha}\right)
$$

and

$$
Z^{\alpha-1}(|V|+|w|+|z|) \leq C\left(\left(\frac{|x|}{|x|+\sqrt{t}}\right)^{\rho}|x|^{-2}+r^{-\frac{2(\alpha-1)}{\alpha}} \boldsymbol{M} h(t, x)\right) .
$$

Estimate (4.13) follows from (4.16), (4.19), (4.20) and (3.15).

Lemma 4.3. Let $\delta>0, T>0$, and $m>\max \{\rho, \alpha \rho\}-2, m \geq 1$. Let $U \in \mathrm{L}^{1}\left((0, T) \times \mathbb{R}^{N}\right)+\mathrm{L}^{\infty}\left((0, T) \times \mathbb{R}^{N}\right)$ satisfy (1.7) for a.a. $0<t<T$ and $x \neq 0$. Let $\chi_{0}$ defined by (4.2) and $\Theta$ by (4.3). Suppose $w_{0} \in \mathrm{L}^{\infty}\left(\mathbb{R}^{N}\right)$ satisfies $\left|w_{0}\right| \leq C \chi_{0}$ and $w \in \mathrm{L}_{\text {loc }}^{1}\left((0, T) \times \mathbb{R}^{N}\right)$ satisfies $|w| \leq C \Theta$ for some constant $C$. It follows that

$$
W(t, x)=: e^{t H} w_{0}+\int_{0}^{t} e^{(t-s) H} \mathcal{M} w(s) \mathrm{d} s,
$$

where $\mathcal{M}$ is given by (1.19), is well defined and $|W| \leq \widetilde{C} h$ with $\widetilde{C}>0$ and $h$ given by (1.23). In particular, $W \in \mathrm{L}^{\frac{2 N}{N-2}}\left((0, T) \times \mathbb{R}^{N}\right)+\mathrm{L}^{\infty}\left((0, T) \times \mathbb{R}^{N}\right)$.

Moreover, $|x|^{-2} W \in \mathrm{L}_{\text {loc }}^{1}\left((0, T) \times \mathbb{R}^{N}\right)$ and

$$
\partial_{t} W-\Delta W-\beta(\alpha+1)|x|^{-2} W=\mathcal{M} w
$$

in $\mathcal{D}^{\prime}\left((0, T) \times \mathbb{R}^{N}\right)$. In addition, $W, \mathcal{M} w \in \mathrm{L}^{\infty}((0, T) \times\{|x|>\varepsilon\})$ for every $\varepsilon>0$.

Proof. The contribution of $w_{0}$ is estimated by (4.6). Next, using (4.13) with $z=0$, and the inequality $\Theta(s, y) \leq$ $s^{\frac{m}{2}}+C^{\prime} \chi_{m}(y)$, we have

$$
\begin{aligned}
|\mathcal{M} w(s, y)| & \leq \boldsymbol{B}_{2} g(s, y)|w(s, y)| \leq C \boldsymbol{B}_{2} g(s, y) \Theta(s, y) \\
& \leq C \boldsymbol{B}_{2} s^{\frac{m}{2}} g(s, y)+C \boldsymbol{B}_{2} C^{\prime} \chi_{m}(y) g(s, y) \\
& \leq C^{\prime \prime} s^{\frac{m}{2}} g(s, y)+C^{\prime \prime} \chi_{m}(y),
\end{aligned}
$$

for some constants $C^{\prime}, C^{\prime \prime}$. (In the last inequality, we used the fact that $g$ defined by (3.15) is bounded on the support of $\chi_{m}$.) Since $h \geq 1$, the contribution of $s^{\frac{m}{2}} g(s, y)$ is estimated by $(3.20)$ with $\varepsilon=0$ and $r=\infty$; and the contribution of $\chi_{m}$ is estimated by (4.6) and the inequality $h(t-s, x) \leq h(t, x)$. It follows that $|W| \leq C h$, and the $\mathrm{L}^{p}$ regularity of $W$ follows from $h(t, x) \leq C+C|x|^{-\eta}$ and $\eta<\frac{N-2}{2}$. The same estimate also shows that 
$|x|^{-2} W \in \mathrm{L}_{\text {loc }}^{1}\left((0, T) \times \mathbb{R}^{N}\right)$. That $W, \mathcal{M} w \in \mathrm{L}^{\infty}((0, T) \times\{|x|>\varepsilon\})$ for every $\varepsilon>0$ follows from $|W| \leq C h$ and from (4.13) with $z=0$.

We now prove (4.22). The term corresponding to $w_{0}$ in (4.21) satisfies the homogeneous equation in $\mathcal{D}^{\prime}((0, T) \times$ $\mathbb{R}^{N}$ ) by Lemma A.4, so we now assume $w_{0}=0$. We fix a function

$$
\zeta \in \mathrm{C}_{\mathrm{c}}^{\infty}\left((0, T) \times \mathbb{R}^{N}\right) .
$$

Next, let $\xi \in \mathrm{C}_{\mathrm{c}}^{\infty}\left(\mathbb{R}^{N}\right)$ satisfy $0 \leq \xi \leq 1, \xi(x)=1$ for $|x| \leq 1$ and $\xi(x)=0$ for $|x| \geq 2$, and set

$$
\psi_{n}(x)=\xi(n x), \quad \theta_{n}(x)=1-\xi\left(\frac{x}{n}\right), \quad \rho_{n}(x)=1-\psi_{n}(x)-\theta_{n}(x),
$$

for $x \in \mathbb{R}^{N}$ and $n \geq 1$. It follows that

$$
\left\|\psi_{n}\right\|_{L^{r}\left(\mathbb{R}^{N}\right)}^{\underset{n \rightarrow \infty}{\longrightarrow} 0}
$$

for all $1 \leq r<\infty$, that $\rho_{n}$ is supported in $\left\{\frac{1}{n} \leq|x| \leq 2 n\right\}$, and that $0 \leq \theta_{n} \leq 1$ and $\theta_{n}(x)=0$ for $|x| \leq n$. We write

$$
W=V_{n}+W_{n}+Z_{n}
$$

where

$$
\begin{aligned}
V_{n} & =\int_{0}^{t} e^{(t-s) H}\left(\psi_{n} \mathcal{M} w\right)(s, \cdot) \mathrm{d} s, \\
W_{n} & =\int_{0}^{t} e^{(t-s) H}\left(\rho_{n} \mathcal{M} w\right)(s, \cdot) \mathrm{d} s, \\
Z_{n} & =\int_{0}^{t} e^{(t-s) H}\left(\theta_{n} \mathcal{M} w\right)(s, \cdot) \mathrm{d} s .
\end{aligned}
$$

Since $|\mathcal{M} w| \leq C g \Theta$ and $\Theta \leq C h$, it follows from (3.17) that

$$
|\mathcal{M} w| \leq C\left(1+|x|^{-\frac{N+2}{2}}\right) .
$$

In particular, we see that $\rho_{n} \mathcal{M} w \in \mathrm{L}^{\infty}\left((0, T), L^{2}\left(\mathbb{R}^{N}\right)\right)$. Applying Lemma A.5, we deduce that

$$
\int_{0}^{T} \int_{\mathbb{R}^{N}} W_{n}\left(-\partial_{t} \zeta-\Delta \zeta-\beta(\alpha+1)|x|^{-2} \zeta\right)=\int_{0}^{T} \int_{\mathbb{R}^{N}} \zeta \rho_{n} \mathcal{M} w
$$

Since $N \geq 3$, the right-hand side of $(4.25)$ is in $\mathrm{L}_{\text {loc }}^{1}\left(\mathbb{R}^{N}\right)$, so that $\zeta \mathcal{M} w \in \mathrm{L}^{1}\left((0, T) \times \mathbb{R}^{N}\right)$. Since $0 \leq \rho_{n} \leq 1$ and $\rho_{n} \rightarrow 1$ a.e., we deduce by dominated convergence that

$$
\int_{0}^{T} \int_{\mathbb{R}^{N}} W_{n}\left(-\partial_{t} \zeta-\Delta \zeta-\beta(\alpha+1)|x|^{-2} \zeta\right) \underset{n \rightarrow \infty}{\longrightarrow} \int_{0}^{T} \int_{\mathbb{R}^{N}} \zeta \mathcal{M} w
$$

Next, we let $0<r<2^{-m} \delta$, so that $1_{\{|y|<r\}} \chi_{m} \equiv 0$ by (4.2); and so it follows from (4.23) that

$$
1_{\{|y|<r\}}|\mathcal{M} w(s, y)| \leq C^{\prime \prime} s^{\frac{m}{2}} 1_{\{|y|<r\}} g(s, y) .
$$


Since $\psi_{n} \leq 1_{\left\{|y|<\frac{2}{n}\right\}}$ and $h \geq 1$, we deduce that for $n \geq \frac{2}{r}$

$$
\psi_{n}|\mathcal{M} w(s, y)| \leq C^{\prime \prime} s^{\frac{m}{2}} 1_{\left\{|y|<\frac{2}{n}\right\}} g(s, y) \leq C^{\prime \prime} s^{\frac{m}{2}} 1_{\left\{|y|<\frac{2}{n}\right\}} g(s, y) h(s, y) .
$$

We fix $0<\varepsilon \leq \varepsilon_{0}$, where $\varepsilon_{0}$ is given by Lemma 3.3, sufficiently small so that

$$
2+\eta+\varepsilon<N
$$

(This is possible, since $\eta<\frac{N-2}{2}$.) It follows from (4.27), (3.20) and (1.23) that

$$
V_{n}(t, x) \leq C n^{-\varepsilon}|x|^{-\varepsilon} h(t, x) \leq C n^{-\varepsilon}|x|^{-\eta-\varepsilon},
$$

on the support of $\zeta$, and we conclude using (4.28) that

$$
\int_{0}^{T} \int_{\mathbb{R}^{N}} V_{n}\left(-\partial_{t} \zeta-\Delta \zeta-\beta(\alpha+1)|x|^{-2} \zeta\right) \underset{n \rightarrow \infty}{\longrightarrow} 0
$$

Moreover, $0 \leq \theta_{n} \leq 1_{\{|x|>n\}}$, so that by Lemma A.3

$$
\sup _{0 \leq t \leq T}\left\|\frac{1}{h(T)} Z_{n}(t)\right\|_{L^{\infty}\left(|x| \leq \frac{n}{2}\right)} \leq C \int_{0}^{T}\left(1+\frac{\sqrt{s}}{n}\right)^{\eta} e^{-\varsigma \frac{n^{2}}{s}} \mathrm{~d} s \underset{n \rightarrow \infty}{\longrightarrow} 0 .
$$

Since $\frac{1}{h(T)} \geq \varepsilon>0$ on the support of $\zeta$, and since the support of $\zeta$ is included in $\left.\left\{|x| \leq \frac{n}{2}\right)\right\}$ for $n$ large, we deduce that

$$
\int_{0}^{T} \int_{\mathbb{R}^{N}} Z_{n}\left(-\partial_{t} \zeta-\Delta \zeta-\beta(\alpha+1)|x|^{-2} \zeta\right) \underset{n \rightarrow \infty}{\longrightarrow} 0
$$

Applying (4.26), (4.29) and (4.30), we see that

$$
\int_{0}^{T} \int_{\mathbb{R}^{N}} W\left(-\partial_{t} \zeta-\Delta \zeta-\beta(\alpha+1)|x|^{-2} \zeta\right)=\int_{0}^{T} \int_{\mathbb{R}^{N}} \zeta \mathcal{M} w
$$

Since $\zeta \in \mathrm{C}_{\mathrm{c}}^{\infty}\left((0, T) \times \mathbb{R}^{N}\right)$ is arbitrary, this proves that $W$ is a solution of $(4.22)$ in $\mathcal{D}^{\prime}\left((0, T) \times \mathbb{R}^{N}\right)$.

\section{Proof of Theorem 1.1}

We prove Theorem 1.1 by using a fixed point argument. We set

$$
w_{0}=u_{0}-U(0),
$$

so that

$$
w_{0} \in \mathrm{L}^{\infty}\left(\mathbb{R}^{N}\right) \quad \text { and } \quad w_{0}=0 \text { on }\{|x|<\delta\}
$$

We let

$$
\boldsymbol{M}=2 \boldsymbol{B}_{1}\left\|w_{0}\right\|_{\mathrm{L}^{\infty}},
$$


where $\boldsymbol{B}_{1}$ is given by (4.6). We fix an integer $m \geq 2, m>\max \{\rho, \alpha \rho\}-2$ (where $\rho>0$ is defined by (1.8)), sufficiently large so that

$$
R_{m}<\frac{1}{4 \boldsymbol{B}_{2}}
$$

where $\boldsymbol{B}_{2}$ is given by (4.13) and $R_{m}$ is given by (3.20) with $\varepsilon=0$. Next, we fix $T \leq S$,

$$
0<T<\frac{1}{4}
$$

sufficiently small so that

$$
\begin{aligned}
e^{-\frac{a_{m+1}^{2}}{2 a t}} \leq t^{\frac{m+1}{2}} \quad \text { for } 0<t & \leq T \\
T^{\frac{1}{2}} \boldsymbol{B}_{1} \boldsymbol{B}_{2} \boldsymbol{A}\left(1+a_{m}^{-\frac{N+2}{2}}\right) & \leq \frac{1}{8}
\end{aligned}
$$

where $\boldsymbol{A}$ is given by Lemma 3.3 and the numbers $a_{j}$ are given by (4.1). Let $\Theta$ be given by $(4.3)$ and let $(\mathcal{E}, \mathrm{d})$ be defined by (4.4)-(4.5) with $\boldsymbol{M}$ given by (5.2). We define $\Phi: \mathcal{E} \mapsto \mathrm{L}^{2}\left((0, T) \times \mathbb{R}^{N}\right)+\mathrm{L}^{\infty}\left((0, T) \times \mathbb{R}^{N}\right)$ by

$$
\Phi(w)(t)=e^{t H} w_{0}+\int_{0}^{t} e^{(t-s) H} \mathcal{M} w(s) \mathrm{d} s .
$$

(Recall that $\Phi(w) \in \mathrm{L}^{\frac{2 N}{N-2}}\left((0, T) \times \mathbb{R}^{N}\right)+\mathrm{L}^{\infty}\left((0, T) \times \mathbb{R}^{N}\right)$ is well defined by Lem. 4.3.) We will show that $\Phi(\mathcal{E}) \subset \mathcal{E}$ and that $\Phi$ has a unique fixed point in $\mathcal{E}$.

Using (5.1), we write $\left|w_{0}\right| \leq\left\|w_{0}\right\|_{L^{\infty} \chi_{0}}$, and we deduce from (4.6), (5.2) and (5.5) that

$$
e^{t H}\left|w_{0}\right| \leq\left\|w_{0}\right\|_{\mathrm{L}^{\infty}} \boldsymbol{B}_{1} h(t)\left(\chi_{1}+e^{-\frac{a_{1}^{2}}{2 a t}}\right) \leq \frac{\boldsymbol{M}}{2} h(t)\left(\chi_{1}+t^{\frac{m}{2}}\right) \leq \frac{\boldsymbol{M}}{2} \Theta .
$$

for all $0<t<T$. If $w \in \mathcal{E}$ then by (4.13) with $z=0$,

$$
|\mathcal{M} w(s)| \leq \boldsymbol{B}_{2} \boldsymbol{M \Theta}(s) g(s)=\boldsymbol{B}_{2} \boldsymbol{M} g(s) h(s)\left(s^{\frac{m}{2}}+\sum_{j=1}^{m} s^{\frac{j-1}{2}} \chi_{j}\right) .
$$

Let now $1 \leq j \leq m$. It follows from (3.17), (4.1) and (5.6) that if $|y|>a_{j}$ and $s<1$, then

$$
g(s, y) h(s, y) \leq \boldsymbol{A}\left(1+|y|^{-\frac{N+2}{2}}\right) \leq \boldsymbol{A}\left(1+a_{j}^{-\frac{N+2}{2}}\right) \leq \boldsymbol{A}\left(1+a_{m}^{-\frac{N+2}{2}}\right) \leq \frac{1}{8 T^{\frac{1}{2}} \boldsymbol{B}_{1} \boldsymbol{B}_{2}} .
$$

We deduce from (5.10) and (4.6) that

$$
\left[e^{(t-s) H} g(s) h(s) \chi_{j}\right](x) \leq \frac{1}{8 T^{\frac{1}{2}} \boldsymbol{B}_{2}} h(t-s, x)\left(e^{-\frac{a_{j+1}^{2}}{2 a(t-s)}}+\chi_{j+1}\right) \leq \frac{1}{8 T^{\frac{1}{2}} \boldsymbol{B}_{2}} h(t, x)\left(e^{-\frac{a_{j+1}^{2}}{2 a t}}+\chi_{j+1}\right)
$$

and so

$$
\int_{0}^{t} e^{(t-s) H} g(s) h(s) s^{\frac{j-1}{2}} \chi_{j} \mathrm{~d} s \leq \frac{1}{8 T^{\frac{1}{2}} \boldsymbol{B}_{2}} h(t, x)\left(e^{-\frac{a_{j+1}^{2}}{2 a t}}+\chi_{j+1}\right) t^{\frac{j+1}{2}}
$$


Since $t<\frac{1}{4}$, we have $\sum_{j=1}^{\infty} t^{\frac{j+1}{2}} \leq \frac{1}{2}$. Thus, (5.11) yields

$$
\int_{0}^{t} e^{(t-s) H} g(s) h(s) \sum_{j=1}^{m} s^{\frac{j-1}{2}} \chi_{j} \mathrm{~d} s \leq \frac{1}{8 T^{\frac{1}{2}} \boldsymbol{B}_{2}} h(t, x)\left(e^{-\frac{a_{m+1}^{2}}{2 a t}}+\sum_{j=1}^{m} t^{\frac{j+1}{2}} \chi_{j+1}\right) .
$$

Using (4.3),(3.20) with $\varepsilon=0$, and (5.12) we obtain

$$
\begin{aligned}
\int_{0}^{t} e^{(t-s) H} \Theta(s) g(s) \mathrm{d} s & \leq h(t)\left(R_{m} t^{\frac{m}{2}}+\frac{1}{8 T^{\frac{1}{2}} \boldsymbol{B}_{2}}\left(e^{-\frac{a_{m+1}^{2}}{2 a t}}+\sum_{j=1}^{m} t^{\frac{j+1}{2}} \chi_{j+1}\right)\right) \\
& =h(t)\left(R_{m} t^{\frac{m}{2}}+\frac{1}{8 T^{\frac{1}{2}} \boldsymbol{B}_{2}}\left(e^{-\frac{a_{m+1}^{2}}{2 a t}}+\sum_{j=2}^{m+1} t^{\frac{j}{2}} \chi_{j}\right)\right) .
\end{aligned}
$$

In particular, since $m \geq 2$, we see that

$$
\int_{0}^{t} e^{(t-s) H} \Theta(s) g(s) \mathrm{d} s \leq C t h(t)
$$

Since $\chi_{1} \geq 0, \chi_{m+1} \leq 1$, and $e^{-\frac{a_{m+1}^{2}}{2 a t}} \leq t^{\frac{m+1}{2}}$ by (5.5), we deduce from (5.13) that

$$
\begin{aligned}
\int_{0}^{t} e^{(t-s) H} \Theta(s) g(s) \mathrm{d} s & \leq h(t)\left(R_{m} t^{\frac{m}{2}}+\frac{1}{8 T^{\frac{1}{2}} \boldsymbol{B}_{2}}\left(t^{\frac{m+1}{2}}+t^{\frac{1}{2}} \sum_{j=1}^{m} t^{\frac{j-1}{2}} \chi_{j}\right)\right) \\
& \leq h(t)\left(R_{m} t^{\frac{m}{2}}+t^{\frac{1}{2}} \frac{1}{8 T^{\frac{1}{2}} \boldsymbol{B}_{2}}\left(t^{\frac{m}{2}}+\sum_{j=1}^{m} t^{\frac{j-1}{2}} \chi_{j}\right)\right) .
\end{aligned}
$$

Applying (5.3) and (5.6) we obtain

$$
\int_{0}^{t} e^{(t-s) H} \Theta(s) g(s) \mathrm{d} s \leq \frac{1}{2 \boldsymbol{B}_{2}} h(t)\left(t^{\frac{m}{2}}+\sum_{j=1}^{m} t^{\frac{j-1}{2}} \chi_{j}\right)=\frac{1}{2 \boldsymbol{B}_{2}} \Theta(t) .
$$

Using the first inequality in (5.9), we deduce that

$$
\left|\int_{0}^{t} e^{(t-s) H} \mathcal{M} w(s) \mathrm{d} s\right| \leq \frac{\boldsymbol{M}}{2} \Theta
$$

With the notation (5.7), it follows from (5.16) and (5.8) that $\Phi(\mathcal{E}) \subset \mathcal{E}$. Moreover, if $w, z \in \mathcal{E}$, we deduce from (4.13) and (4.5) that

$$
|\mathcal{M} w(s)-\mathcal{M} z(s)| \leq \boldsymbol{B}_{2}|w(s)-z(s)| g(s) \leq \boldsymbol{B}_{2} d(w, z) \Theta(s) g(s) .
$$

Using again (5.15) we obtain

$$
|\Phi(w)(t)-\Phi(z)(t)| \leq \int_{0}^{t} e^{(t-s) H}|\mathcal{M} w(s)-\mathcal{M} z(s)| \mathrm{d} s \leq \frac{1}{2} \Theta(t) d(w, z) .
$$


Therefore, $\mathrm{d}(\Phi(w), \Phi(z)) \leq \frac{1}{2} d(w, z)$, so that $\Phi$ has a unique fixed point $w \in \mathcal{E}$. We deduce from Lemma 4.3 that

$$
\partial_{t} w-\Delta w-\beta(\alpha+1)|x|^{-2} w=\mathcal{M} w
$$

in $\mathcal{D}^{\prime}\left((0, T) \times \mathbb{R}^{N}\right)$. Therefore,

$$
\partial_{t} w-\Delta w=|U+w|^{\alpha}(U+w)-|U|^{\alpha} U
$$

in $\mathcal{D}^{\prime}\left((0, T) \times \mathbb{R}^{N}\right)$, by (1.19). Since the right-hand side of the above equation is in $\mathrm{L}_{\text {loc }}^{\infty}\left((0, T) \times\left(\mathbb{R}^{N} \backslash\{0\}\right)\right)$ by $w \in \mathcal{E}$ and (1.7), it follows from Lemma A.6 that $w \in \mathrm{C}\left((0, T) \times\left(\mathbb{R}^{N} \backslash\{0\}\right)\right)$.

We now set

$$
u=U+w,
$$

so that $u \in \mathrm{L}_{\text {loc }}^{\alpha+1}\left((0, T) \times \mathbb{R}^{N}\right) \cap \mathrm{C}\left((0, T) \times\left(\mathbb{R}^{N} \backslash\{0\}\right)\right)$ is a solution of $(1.10)$ in $\mathcal{D}^{\prime}\left((0, T) \times \mathbb{R}^{N}\right)$. Estimate $(1.12)$ follows from (5.18) and $w \in \mathcal{E}$. Since $w \in \mathcal{E}$, we have $|\mathcal{M} w| \leq C \Theta g$ by (4.13) with $z=0$, so it follows from (5.14) that

$$
\left|w(t)-e^{t H} w_{0}\right| \leq C t h(t)
$$

Finally, since $u \in \mathrm{C}\left((0, T) \times\left(\mathbb{R}^{N} \backslash\{0\}\right)\right)$, we see that $u(t, x)$ is defined for all $0<t<T$ and $x \neq 0$, and using (1.12), we deduce that $u(t) \in \mathrm{L}_{\mathrm{loc}}^{1}\left(\mathbb{R}^{N}\right)$ for all $0<t<T$. Moreover, given $R>0$,

$$
\begin{gathered}
\left\|u(t)-u_{0}\right\|_{\mathrm{L}^{1}(\{|x|<R\})} \leq \\
+\|(t)-U(0)\|_{\mathrm{L}^{1}(\{|x|<R\})}+\left\|w(t)-e^{t H} w_{0}\right\|_{\mathrm{L}^{1}(\{|x|<R\})} \\
+\left\|e^{t H} w_{0}-w_{0}\right\|_{\mathrm{L}^{1}(\{|x|<R\}) \underset{t \rightarrow 0}{\longrightarrow} 0}
\end{gathered}
$$

by (1.9), (5.19), and Lemma A.4. This proves (1.10) and completes the proof of Theorem 1.1.

\section{Singular, RADiAlly SYMMETRIC, STATIONARY SOLUTIONS}

Let

$$
N \geq 3 \text { and } \frac{2}{N-2}<\alpha<\frac{4}{N-2}
$$

and let $\beta>0$ be defined by (1.4). We study radially symmetric, possibly sign-changing stationary solutions of (1.1). Such solutions satisfy the ODE

$$
u^{\prime \prime}+\frac{N-1}{r} u^{\prime}+|u|^{\alpha} u=0
$$

It follows easily from energy arguments that any solution of (6.2) on some interval $(a, b)$ with $0 \leq a<b \leq \infty$ can be extended to a solution on $(0, \infty)$.

All the positive solutions of (6.2) are known, see ([21], Prop. 3.1). They consist of the solution $\beta^{\frac{1}{\alpha}}|x|^{-\frac{2}{\alpha}}$ and a one-parameter family $\left(u_{\lambda}\right)_{\lambda>0} \subset \mathrm{C}^{2}(0, \infty)$ of singular solutions satisfying $r^{\frac{2}{\alpha}} u_{\lambda}(r) \rightarrow \beta^{\frac{1}{\alpha}}$ as $r \rightarrow 0$ and $r^{N-2} u_{\lambda}(x) \rightarrow \lambda$ as $r \rightarrow \infty$. The negative solutions of (6.2) are therefore $-\beta^{\frac{1}{\alpha}}|x|^{-\frac{2}{\alpha}}$ and $\left(-u_{\lambda}\right)_{\lambda>0}$. All other solutions of (6.2) are therefore sign-changing, and they consist of the one-parameter family of regular solutions, i.e. the solutions of (6.2) with the initial conditions $u(0)=c \in \mathbb{R}, u^{\prime}(0)=0$ and the (two-parameter family) of sign-changing, singular solutions. More precisely, we have the following result. 
Proposition 6.1. Assume (6.1) and let $\beta>0$ be defined by (1.4). If $u \in \mathrm{C}^{2}(0, \infty), u \not \equiv 0$, is a solution of (6.2), then the following properties hold.

(i) $|u(r)| \leq C r^{-\left(N-2-\frac{2}{\alpha}\right)}$ for $r>1$. In addition, either $u(r)$ has a finite limit as $r \rightarrow 0$, or else $r^{\frac{2}{\alpha}} u(r) \rightarrow \pm \beta^{\frac{1}{\alpha}}$ as $r \rightarrow 0$. In the first case, $u^{\prime}(r) \rightarrow 0$ as $r \rightarrow 0$, and in the second case $r^{\frac{2}{\alpha}+1} u^{\prime}(r) \rightarrow \mp \frac{2}{\alpha} \beta^{\frac{1}{\alpha}}$.

(ii) $u$ satisfies one of the following properties.

(a) $u$ is a regular solution of $(6.2)$, i.e. $u \in \mathrm{C}^{2}([0, \infty))$ and $u^{\prime}(0)=0$. In this case, $u$ oscillates indefinitely as $r \rightarrow \infty$.

(b) $u$ is a constant-sign, singular solution of (6.2), i.e. either $u= \pm \beta^{\frac{1}{\alpha}} r^{-\frac{2}{\alpha}}$ or else $u= \pm u_{\lambda}$ for some $\lambda>0$ where $u_{\lambda}$ is as defined above.

(c) $u$ is a singular, sign-changing solution. In this case, $r^{\frac{2}{\alpha}} u(r) \rightarrow \pm \beta^{\frac{1}{\alpha}}$ as $r \rightarrow 0$, and $u$ oscillates indefinitely as $r \rightarrow \infty$.

(iii) $u$ satisfies (iic) if and only if there exists $r_{0}>0$ such that

$$
\frac{r_{0}^{2}}{2}\left|u^{\prime}\left(r_{0}\right)\right|^{2}+\frac{2}{\alpha} r_{0} u\left(r_{0}\right) u^{\prime}\left(r_{0}\right)+\frac{1}{\alpha+2} r_{0}^{2}\left|u\left(r_{0}\right)\right|^{\alpha+2}+\frac{4-(N-2) \alpha}{\alpha^{2}}\left|u\left(r_{0}\right)\right|^{2}=0 .
$$

(iv) Let $U \in \mathrm{C}^{2}\left(\mathbb{R}^{N} \backslash\{0\}\right)$ be defined by $U(x)=u(|x|)$ for $x \neq 0$. It follows that $U \in \mathrm{L}_{\text {loc }}^{\alpha+1}\left(\mathbb{R}^{N}\right)$ and that $U$ is a solution of

$$
-\Delta U=|U|^{\alpha} U
$$

in $\mathcal{D}^{\prime}\left(\mathbb{R}^{N}\right)$.

Proof. Given a solution $u \in C^{2}(0, \infty)$ of $(6.2)$, we set (see [11], Eq. (6))

$$
u(r)=r^{-\frac{2}{\alpha}} v(s), \quad s=-\log r,
$$

so that $v \in \mathrm{C}^{2}(\mathbb{R})$ satisfies

$$
v^{\prime \prime}+\gamma v^{\prime}-\beta v+|v|^{\alpha} v=0
$$

for all $s \in \mathbb{R}$, where $\beta$ is given by (1.4) and

$$
\gamma=\frac{4}{\alpha}-N+2>0
$$

(Note that we use (6.1) to obtain $\beta, \gamma>0$.) Equation (6.6) has the stationary solutions 0 and $\pm \beta^{\frac{1}{\alpha}}$, corresponding to the solutions $u(r) \equiv 0$ and $u(r) \equiv \pm \beta^{\frac{1}{\alpha}} r^{-\frac{2}{\alpha}}$ of (6.2). We now suppose that $v$ is not a stationary solution of (6.6). Setting

$$
F\left(v, v^{\prime}\right)=\frac{1}{2}\left|v^{\prime}\right|^{2}+\frac{1}{\alpha+2}|v|^{\alpha+2}-\frac{\beta}{2}|v|^{2}
$$

we see that

$$
f(s)=: F\left(v(s), v^{\prime}(s)\right)
$$

satisfies

$$
f^{\prime}(s)+\gamma\left|v^{\prime}(s)\right|^{2}=0
$$


We first note that, since $v$ is not a stationary solution, $\int_{s_{0}-1}^{s_{0}}\left|v^{\prime}\right|^{2}>0$ for all $s<s_{0}$, and it follows from (6.10), that

$$
f\left(s_{1}\right)>f\left(s_{2}\right) \text { for all } s_{1}<s_{2}
$$

Next,

$$
F\left(v, v^{\prime}\right) \geq F\left(\beta^{\frac{1}{\alpha}}, 0\right)=-\frac{\alpha}{2(\alpha+2)} \beta^{\frac{\alpha+2}{\alpha}}=: F_{\star} .
$$

It follows that $f$ is bounded from below, so that $f(s)$ decreases to a limit $f_{\infty}$ as $s \rightarrow \infty$. We deduce easily that

$$
\sup _{s \geq 0}\left(|v(s)|+\left|v^{\prime}(s)\right|\right)<\infty
$$

and

$$
\gamma \int_{s}^{\infty}\left|v^{\prime}(\tau)\right|^{2} \mathrm{~d} \tau=f(s)-f_{\infty}<\infty
$$

Using (6.13)-(6.14), it follows by standard arguments that

$$
v^{\prime}(s) \underset{s \rightarrow \infty}{\longrightarrow} 0, \quad v(s) \underset{s \rightarrow \infty}{\longrightarrow} \ell, \quad \text { with } \quad \ell=0 \quad \text { or } \quad \ell= \pm \beta^{\frac{1}{\alpha}}
$$

We now proceed in seven steps.

Step 1. The case $\ell=0$ in (6.15). This corresponds to the case where $u(r)$ has a finite limit as $r \rightarrow 0$, and then $u^{\prime}(r) \rightarrow 0$ as $r \rightarrow 0$. (See [21], Eq. (2.4).) Note that in this case, $f(s) \rightarrow 0$ as $s \rightarrow \infty$, so that by (6.11)

$$
f(s)>0, \quad s \in \mathbb{R} .
$$

Step 2. The case $\ell= \pm \beta^{\frac{1}{\alpha}}$ in (6.15). This means that

$$
r^{\frac{2}{\alpha}} u(r) \underset{r \rightarrow 0}{\longrightarrow} \pm \beta^{\frac{1}{\alpha}}
$$

In addition,

$$
r^{\frac{2}{\alpha}+1} u^{\prime}(r)=-\frac{2}{\alpha} v(s)-v^{\prime}(s) \underset{r \rightarrow 0}{\longrightarrow} \mp \frac{2}{\alpha} \beta^{\frac{1}{\alpha}}
$$

Moreover,

$$
f(s) \underset{s \rightarrow \infty}{\longrightarrow} F_{\star}<0 .
$$

Step 3. We prove Property (i). We first prove that $|u(r)| \leq C r^{-\left(N-2-\frac{2}{\alpha}\right)}$ for $r>1$. In terms of $v$, this means

$$
|v(s)| \leq C e^{-\gamma s}, \quad s \leq 0 .
$$


Integrating $(6.10)$ on $(s, 0)$ we obtain

$$
\left|v^{\prime}\right|^{2}=\beta|v|^{2}-\frac{2}{\alpha+2}|v|^{\alpha+2}+2 F\left(v(0), v^{\prime}(0)\right)+2 \gamma \int_{s}^{0}\left|v^{\prime}(\sigma)\right|^{2} \mathrm{~d} \sigma \leq C+2 \gamma \int_{s}^{0}\left|v^{\prime}(\sigma)\right|^{2} \mathrm{~d} \sigma,
$$

for some constant $C$ so that, by Gronwall's inequality, $\left|v^{\prime}(s)\right| \leq C e^{-\gamma s}$ for $s<0$. Estimate (6.20) follows by integration. The part of Property (i) concerning the behavior of $u(r)$ as $r \rightarrow 0$ follows from Steps 1 and 2 .

Step 4. We prove that if $f\left(s_{0}\right) \geq 0$ for some $s_{0} \in \mathbb{R}$, then $v$ oscillates indefinitely as $s \rightarrow-\infty$. Suppose by contradiction that (for instance) $v(s)>0$ for $s<s_{1}$ with $s_{1}<s_{0}$. This means that $u(r)>0$ for $r$ large. It follows from Lemma 2.0 of [21] that $u(r)>0$ for all $r>0$. Applying now ([21], Prop. 3.1), we deduce that $u$ is either the solution $\beta^{\frac{1}{\alpha}}|x|^{-\frac{2}{\alpha}}$ or a solution $u_{\lambda}$ for some $\lambda>0$. The first case corresponds to $v(s) \equiv \beta^{\frac{1}{\alpha}}$, so that $f(s) \equiv F_{\star}<0$, which is absurd. In the second case, $u(r) \leq c r^{-(N-2)}$, so that $v(s) \rightarrow 0$ as $s \rightarrow-\infty$. Therefore, $v^{\prime}\left(s_{n}\right) \rightarrow 0$ for some sequence $s_{n} \rightarrow-\infty$, so that $f\left(s_{n}\right) \rightarrow 0$ as $n \rightarrow \infty$. On the other hand, it follows from (6.11) that for $n$ large, $f\left(s_{n}\right)>f\left(s_{0}-1\right)>0$. This is again absurd.

Step 5. We prove Property (ii). We consider three cases.

- If $f(s)>0$ for all $s \in \mathbb{R}$, then $v$ oscillates indefinitely as $s \rightarrow-\infty$ by Step 4 ; and so, $u$ oscillates indefinitely as $r \rightarrow \infty$. Moreover, it follows from (6.19) that $\ell=0$ in (6.15). By Step 1, this implies that $u$ satisfies (iia).

- If $f(s)<0$ for all $s \in \mathbb{R}$, then $\ell= \pm \beta^{\frac{1}{\alpha}}$ in (6.15). Indeed, the case $\ell=0$ is ruled out by (6.16). Moreover, $v$ cannot vanish, for if $v(s)=0$, then $f(s)>0$. Therefore, $u$ is a constant-sign singular solution of (6.2), and it follows from ([21], Prop. 3.1) that $u$ satisfies (iib).

- If $f\left(s_{0}\right)=0$ for some $s_{0} \in \mathbb{R}$, then $v$ oscillates indefinitely as $s \rightarrow-\infty$ by Step 4 ; and so, $u$ oscillates indefinitely as $r \rightarrow \infty$. In addition, it follows from (6.11) that $f(s)<0$ for $s>s_{0}$. Therefore, $\ell= \pm \beta^{\frac{1}{\alpha}}$ in (6.15). Indeed, the case $\ell=0$ is ruled out by (6.16). Therefore, $u$ is a singular solution of (6.2) that oscillates indefinitely as $r \rightarrow \infty$. This means that $u$ satisfies (iic).

Step 6. We prove Property (iii). The discussion in Step 5 shows that $u$ satisfies (iic) if and only $f\left(s_{0}\right)=0$ for some $s_{0} \in \mathbb{R}$. This is equivalent to (6.3) with $r_{0}=e^{-s_{0}}$. Note that $f$ is decreasing by (6.11), so that $f$ can vanish at most for one value of $s$, and therefore (6.3) can be satisfied at most for one value of $r_{0}$.

Step 7. We prove Property (iv). This is well known if $u$ is a regular solution. In the general case, $|U(x)| \leq$ $C\left(1+|x|^{-\frac{2}{\alpha}}\right)$ by Property (i); and so $U \in \mathrm{L}_{\text {loc }}^{\alpha+1}\left(\mathbb{R}^{N}\right)$ by $(6.1)$. Let now $\varphi \in \mathrm{C}_{\mathrm{c}}^{\infty}\left(\mathbb{R}^{N}\right)$. Since $U \in \mathrm{L}_{\text {loc }}^{\alpha+1}\left(\mathbb{R}^{N}\right)$, we see that

$$
\left|\int_{\{|x|<\varepsilon\}}\left(U \Delta \varphi+|U|^{\alpha} U \varphi\right)\right| \leq C \int_{\{|x|<\varepsilon\}}\left(|U|+|U|^{\alpha+1}\right) \underset{\varepsilon \downarrow 0}{\longrightarrow} 0
$$

On the other hand, $U$ is a classical solution of (6.4) on $\mathbb{R}^{N} \backslash\{0\}$, so that integration by parts yields

$$
\int_{\{|x|>\varepsilon\}}\left(U \Delta \varphi+|U|^{\alpha} U \varphi\right)=-\int_{\{|x|=\varepsilon\}}\left(U \frac{\partial \varphi}{\partial r}-\varphi \frac{\partial U}{\partial r}\right) .
$$

Since $\varepsilon^{N-1}\left(|u(\varepsilon)|+\left|u^{\prime}(\varepsilon)\right|\right) \rightarrow 0$ as $\varepsilon \rightarrow 0$ by Property (i), we deduce that

$$
\int_{\{|x|>\varepsilon\}}\left(U \Delta \varphi+|U|^{\alpha} U \varphi\right) \underset{\varepsilon \rightarrow 0}{\longrightarrow} 0 .
$$

Estimates (6.22) and (6.23) imply

$$
\int_{\mathbb{R}^{N}}\left(U \Delta \varphi+|U|^{\alpha} U \varphi\right)=0
$$


Since $\varphi \in \mathrm{C}_{\mathrm{c}}^{\infty}\left(\mathbb{R}^{N}\right)$ is arbitrary, we see that $U$ is a solution of $(6.4)$ in $\mathcal{D}^{\prime}\left(\mathbb{R}^{N}\right)$.

Corollary 6.2. Assuming (6.1), there exists a two-parameter family of radially symmetric, sign-changing, stationary solutions $U \in \mathrm{C}^{2}\left(\mathbb{R}^{N} \backslash\{0\}\right) \cap \mathrm{L}_{\text {loc }}^{\alpha+1}\left(\mathbb{R}^{N}\right)$ of (1.1) in the sense of distributions, which are singular at $x=0$. These solutions satisfy $|U(x)| \leq C|x|^{-\left(N-2-\frac{2}{\alpha}\right)}$ for $|x|>1$ and oscillate indefinitely as $|x| \rightarrow \infty$.

Proof. Applying Proposition 6.1, we need only show that there is a two-parameter family of solutions of (6.2) that satisfy (6.3) for some $r_{0}>0$ (depending on the solution). To see this, consider any $r_{0}>0$ and $0<a<$ $\left(\frac{\alpha+2}{2}\right)^{\frac{1}{\alpha}}$. Let $b>\frac{2 a}{\alpha}$ be defined by

$$
\frac{1}{2}\left(b-\frac{2 a}{\alpha}\right)^{2}=a^{2} \frac{(N-2) \alpha-2}{\alpha^{2}(\alpha+2)}\left[\alpha+2-2 a^{\alpha}\right] .
$$

If $u$ is the solution of (6.2) defined by $u\left(r_{0}\right)=a \beta^{\frac{1}{\alpha}} r_{0}^{-\frac{2}{\alpha}}$ and $u^{\prime}\left(r_{0}\right)=-b \beta^{\frac{1}{\alpha}} r_{0}^{-\frac{2}{\alpha}-1}$, then (6.3) is satisfied. It remains to prove that two different choices of $\left(r_{0}, a\right)$ yield two different solutions of (6.2). Suppose two choices $r_{0}^{1}, r_{0}^{2}>0$ and $a^{1}, a^{2} \in\left(0,\left(\frac{\alpha+2}{2}\right)^{\frac{1}{\alpha}}\right)$ produce the same solution $u$ of $(6.2)$. With the notation used in the proof of Proposition 6.1, we have $f\left(s_{0}^{1}\right)=f\left(s_{0}^{2}\right)$, where $s_{0}^{j}=-\log r_{0}^{j}, j=1$, 2. (See Step 6 of the proof of Prop. 6.1.) Note that $u(r) \not \equiv 0$ since $a^{1} \neq 0$, and that $u(r) \not \equiv \beta^{\frac{1}{\alpha}} r^{-\frac{2}{\alpha}}$ since $u$ is sign-changing. Therefore, it follows from (6.11) that $s_{0}^{1}=s_{0}^{2}$, so that $r_{0}^{1}=r_{0}^{2}$. Since $u\left(r_{0}^{j}\right)=a^{j} \beta^{\frac{1}{\alpha}}\left(r_{0}^{j}\right)^{-\frac{2}{\alpha}}, j=1,2$, we conclude that also $a^{1}=a^{2}$. This completes the proof.

\section{Proof of Theorems 1.3 AND 1.4}

In this section, we give the proof of Theorems 1.3 and 1.4, which are consequences of Theorem 1.1. We begin with the following proposition, which provides refined estimates for the behavior at the origin of both the radially symmetric singular stationary solutions of (1.1) and of the profiles of singular, radially symmetric, self-similar solutions of (1.1).

Proposition 7.1. Assume (1.2). Let $a \in \mathbb{R}$ and $u \in \mathrm{C}^{2}(0, \infty)$ a solution of

$$
u^{\prime \prime}+\left(\frac{N-1}{r}+\frac{a r}{2}\right) u^{\prime}+\frac{a}{\alpha} u+|u|^{\alpha} u=0 .
$$

If

$$
r^{\frac{2}{\alpha}} u(r) \underset{r \rightarrow 0}{\longrightarrow} \beta^{\frac{1}{\alpha}}
$$

where $\beta$ is defined by (1.4), then there exists a constant $C$ such that

$$
\left|r^{\frac{2}{\alpha}} u(r)-\beta^{\frac{1}{\alpha}}\right| \leq C r^{\rho},
$$

for all $0<r \leq 1$, where $\rho>0$ is defined by (1.8).

Proof. Setting

$$
u(r)=r^{-\frac{2}{\alpha}} v(s), \quad s=-\log r,
$$

the equation for $v$ is

$$
v^{\prime \prime}+\left(\gamma-a e^{-2 s}\right) v^{\prime}-\beta v+|v|^{\alpha} v=0
$$


where

$$
\gamma=\frac{4}{\alpha}-N+2>0
$$

Let

$$
v=\beta^{\frac{1}{\alpha}}+z
$$

and set

$$
g(s)=|s|^{\alpha} s, \quad \varphi(s)=g\left(\beta^{\frac{1}{\alpha}}+s\right)-g\left(\beta^{\frac{1}{\alpha}}\right)-g^{\prime}\left(\beta^{\frac{1}{\alpha}}\right) s .
$$

It follows that

$$
z^{\prime \prime}+\gamma z^{\prime}+\alpha \beta z=h,
$$

where

$$
h=a e^{-2 s} z^{\prime}-\varphi(z) .
$$

Note that the fundamental solutions of the linear equation $z^{\prime \prime}+\gamma z^{\prime}+\alpha \beta z=0$ are

$$
\phi_{1}(s)=e^{-2 \mu_{1} s}, \quad \phi_{2}(s)=e^{-2 \mu_{2} s},
$$

where $\mu_{1}, \mu_{2}$ are given by Lemma 2.1. By the variation of the parameter formula, we deduce that

$$
z(s)=\left(C_{1}+\frac{1}{2\left(\mu_{2}-\mu_{1}\right)} \int_{s_{0}}^{s} e^{2 \mu_{1} \sigma} h \mathrm{~d} \sigma\right) e^{-2 \mu_{1} s}+\left(C_{2}-\frac{1}{2\left(\mu_{2}-\mu_{1}\right)} \int_{s_{0}}^{s} e^{2 \mu_{2} \sigma} h \mathrm{~d} \sigma\right) e^{-2 \mu_{2} s},
$$

for some appropriate constants $C_{1}, C_{2}$, and

$$
z^{\prime}(s)=-2 \mu_{1}\left(C_{1}+\frac{1}{2\left(\mu_{2}-\mu_{1}\right)} \int_{s_{0}}^{s} e^{2 \mu_{1} \sigma} h \mathrm{~d} \sigma\right) e^{-2 \mu_{1} s}-2 \mu_{2}\left(C_{2}-\frac{1}{2\left(\mu_{2}-\mu_{1}\right)} \int_{s_{0}}^{s} e^{2 \mu_{2} \sigma} h \mathrm{~d} \sigma\right) e^{-2 \mu_{2} s} .
$$

We first prove that there exists $\varepsilon>0$ such that

$$
|z(s)|+\left|z^{\prime}(s)\right| \leq C e^{-\varepsilon s}, \quad s \geq 0 .
$$

To see this, we fix $\varepsilon>0$ sufficiently small so that

$$
\gamma-4 \varepsilon>0, \quad \alpha \beta+4 \varepsilon^{2}-2 \gamma \varepsilon>0,
$$

and we set

$$
\eta_{1}=\frac{\gamma-4 \varepsilon}{2}>0, \quad \eta_{2}=\frac{\alpha \beta+4 \varepsilon^{2}-2 \gamma \varepsilon}{2}>0 .
$$

Setting

$$
z(s)=e^{-2 \varepsilon s} \psi(s),
$$


we obtain for $\psi$ the equation

$$
\psi^{\prime \prime}+2 \eta_{1} \psi^{\prime}+2 \eta_{2} \psi=a e^{-2 s}\left(\psi^{\prime}-2 \varepsilon \psi\right)-e^{2 \varepsilon s} \varphi(z) .
$$

Multiplying by $\psi^{\prime}$, we obtain

$$
\frac{\mathrm{d}}{\mathrm{d} s}\left(\frac{1}{2}\left|\psi^{\prime}\right|^{2}+\eta_{2} \psi^{2}\right)+\left(2 \eta_{1}-a e^{-2 s}\right)\left|\psi^{\prime}\right|^{2}=-2 \varepsilon a e^{-2 s} \psi \psi^{\prime}-e^{2 \varepsilon s} \varphi(z) \psi^{\prime} .
$$

Now

$$
-2 \varepsilon a e^{-2 s} \psi \psi^{\prime}-e^{2 \varepsilon s} \varphi(z) \psi^{\prime} \leq \varepsilon a e^{-2 s} \psi^{2}+\varepsilon a e^{-2 s}\left|\psi^{\prime}\right|^{2}+\eta_{1}\left|\psi^{\prime}\right|^{2}+\frac{1}{4 \eta_{1}}\left(e^{2 \varepsilon s} \varphi(z)\right)^{2} .
$$

We first choose $s_{0}$ sufficiently large so that

$$
a e^{-2 s} \leq \eta_{2} \quad \text { and } \quad \varepsilon a e^{-2 s} \leq \eta_{1}-a e^{-2 s} \text { for } s \geq s_{0},
$$

and we obtain

$$
\frac{\mathrm{d}}{\mathrm{d} s}\left(\frac{1}{2}\left|\psi^{\prime}\right|^{2}+\eta_{2} \psi^{2}\right) \leq \varepsilon \eta_{2} \psi^{2}+\frac{1}{4 \eta_{1}}\left(e^{2 \varepsilon s} \varphi(z)\right)^{2} .
$$

Next we observe that there exists a constant $A$ such that

$$
|\varphi(z)| \leq A z^{2}, \quad|z| \leq 1 .
$$

Note that by (7.2) $|z(s)| \rightarrow 0$ as $s \rightarrow \infty$, so that by possibly choosing $s_{0}$ larger we have $|z(s)| \leq 1$ for $s \geq s_{0}$. Therefore

$$
\left|e^{2 \varepsilon s} \varphi(z)\right| \leq A e^{2 \varepsilon s} z^{2}=A|z||\psi| .
$$

Thus we see that

$$
\frac{\mathrm{d}}{\mathrm{d} s}\left(\frac{1}{2}\left|\psi^{\prime}\right|^{2}+\eta_{2} \psi^{2}\right) \leq \varepsilon \eta_{2} \psi^{2}+\frac{A^{2} z^{2}}{4 \eta_{1}} \psi^{2} .
$$

By choosing $s_{0}$ possibly larger, we deduce from (7.2) that

$$
\frac{A^{2} z^{2}}{4 \eta_{1}} \leq \varepsilon \eta_{2} \quad s \geq s_{0}
$$

and we conclude that

$$
\frac{\mathrm{d}}{\mathrm{d} s}\left(\frac{1}{2}\left|\psi^{\prime}\right|^{2}+\eta_{2} \psi^{2}\right) \leq 2 \varepsilon \eta_{2} \psi^{2} \leq 2 \varepsilon\left(\frac{1}{2}\left|\psi^{\prime}\right|^{2}+\eta_{2} \psi^{2}\right),
$$

for $s \geq s_{0}$. Applying Gronwall's inequality, we deduce that

$$
|\psi|+\left|\psi^{\prime}\right| \leq C e^{\varepsilon s},
$$

Using (7.10), the claim (7.7) follows. 
We complete the proof by using formulas (7.5)-(7.6) and a bootstrap argument. We note that $\rho=2 \mu_{1}$ by Lemma 2.1, so that (7.3) is proved if

$$
|z(s)|+\left|z^{\prime}(s)\right| \leq C e^{-2 \mu_{1} s}, \quad s \geq 0
$$

Suppose that

$$
|z(s)|+\left|z^{\prime}(s)\right| \leq C e^{-\nu s}, \quad s \geq 0,
$$

for some $\nu>0$. It follows from (7.4), (7.13) and (7.11) that

$$
|h| \leq C e^{-\widetilde{\nu}}
$$

where

$$
\widetilde{\nu}=\min \{\nu+2,2 \nu\}=\nu+\min \{2, \nu\}>\nu .
$$

It is not difficult to deduce from (7.5)-(7.6) that

$$
|z(s)|+\left|z^{\prime}(s)\right| \leq \begin{cases}C e^{-\widetilde{\nu}} & \widetilde{\nu}<2 \mu_{1}, \\ C(1+s) e^{-2 \mu_{1}} & \widetilde{\nu}=2 \mu_{1}, \\ C e^{-2 \mu_{1}} & \widetilde{\nu}>2 \mu_{1} .\end{cases}
$$

We now conclude as follows. It follows from (7.7) that (7.13) holds for $\widetilde{\nu}=\varepsilon>0$. If $\varepsilon>2 \mu_{1}$, then the estimate (7.12) follows from (7.15). If $\varepsilon \leq 2 \mu_{1}$, then it follows from (7.15) that (7.13) holds with

$$
\nu=\varepsilon+\frac{1}{2} \min \{2, \varepsilon\}
$$

We now can iterate the above argument. In at most $\ell$ steps, where

$$
\frac{\ell}{2} \min \{2, \varepsilon\}>2 \mu_{1},
$$

we obtain the estimate (7.12).

We are now in a position to prove Theorem 1.3.

Proof of Theorem 1.3. Let $U$ be a radially symmetric, stationary solution of (1.1) that is singular at $x=0$. It follows that $U(x)=u(|x|)$ where $u \in C^{2}(0, \infty), u \not \equiv 0$, is a solution of (6.2) which is singular at $r=0$. We deduce from Proposition 6.1 (ii) that $r^{\frac{2}{\alpha}} u(r) \rightarrow \pm \beta^{\frac{1}{\alpha}}$ as $r \rightarrow 0$. Therefore, we may apply Proposition 7.1 with $a=0$, and we obtain that $u$ satisfies (7.3). Since $u$ is bounded as $r \rightarrow \infty$ by Proposition 6.1 (i), it follows that $U$ satisfies (1.6), and also (1.7) for all $0<t<1$ and $x \neq 0$, while (1.9) is trivial with $U_{0}=U$. Since $\partial_{t} U-\Delta U-|U|^{\alpha} U=0$ in $\mathcal{D}^{\prime}\left((0, T) \times \mathbb{R}^{N}\right)$ by Proposition 6.1 (iv), the result now follows by applying Theorem 1.1 with $S=1$.

For the proof of Theorem 1.4, we will use the following proposition.

Proposition 7.2. Assume (6.1) and let $f \in \mathrm{C}^{2}(0, \infty)$ be a solution of the equation (1.5). It follows that

$$
\ell=\lim _{r \rightarrow 0} r^{\frac{2}{\alpha}} f(r)
$$


exists and either $\ell=0$ or else $\ell= \pm \beta^{\frac{1}{\alpha}}$, where $\beta$ is defined by (1.4); and

$$
\lim _{r \rightarrow 0} r^{1+\frac{2}{\alpha}} f^{\prime}(r)=-\frac{2}{\alpha} \ell
$$

Moreover,

$$
\mu=\lim _{r \rightarrow \infty} r^{\frac{2}{\alpha}} f(r)
$$

exists and is finite. In addition, if $U \in \mathrm{C}^{2}\left((0, \infty) \times\left(\mathbb{R}^{N} \backslash\{0\}\right)\right)$ is defined by

$$
U(t, x)=t^{-\frac{1}{\alpha}} f\left(\frac{|x|}{\sqrt{t}}\right), \quad t>0, x \neq 0,
$$

then the following properties hold.

(i) $U \in \mathrm{L}_{\text {loc }}^{\alpha+1}\left((0, S) \times \mathbb{R}^{N}\right) \cap \mathrm{C}\left((0, S) \times\left(\mathbb{R}^{N} \backslash\{0\}\right)\right)$ is a solution of $(1.1)$ in $\mathcal{D}^{\prime}\left((0, T) \times \mathbb{R}^{N}\right)$.

(ii) If $U_{0}(x)=\mu|x|^{-\frac{2}{\alpha}}$, then

$$
U(t) \underset{t \rightarrow 0}{\longrightarrow} U_{0}
$$

in $\mathrm{L}_{\text {loc }}^{1}\left(\mathbb{R}^{N}\right)$.

(iii) If (1.2) holds and $\ell=\beta^{\frac{1}{\alpha}}$ in (7.16), then $U$ satisfies (1.7) for all $t>0$ and $x \neq 0$.

Moreover, there exist a sequence $\left(\mu_{n}\right)_{n \geq 1} \subset(0, \infty), \mu_{n} \rightarrow \infty$ and a sequence $\left(f_{n}\right)_{n \geq 1}$ of sign-changing solutions of the equation (1.5) for which $\lim _{r \rightarrow 0} r^{\frac{2}{\alpha}} f_{n}(r)=\beta^{\frac{1}{\alpha}}$ and $\lim _{r \rightarrow \infty} r^{\frac{2}{\alpha}} f_{n}(r)=\mu_{n}$.

Proof. The existence of the limit (7.16) follows from Propositions 3.2 and 3.3, and formula (1.9) in [22], then the limit (7.17) follows from Proposition 3.1 (i) in [22]. The existence of the limit (7.18) follows from Proposition 2.4 and formula (1.9) in [22]. Next, we show that $V(x)=U(1, x)=f(|x|)$ is a solution of

$$
\Delta V+\frac{1}{2} x \cdot \nabla V+\frac{1}{\alpha} V+|V|^{\alpha} V=0
$$

in $\mathcal{D}^{\prime}\left(\mathbb{R}^{N}\right)$. Let $\varphi \in \mathrm{C}_{\mathrm{c}}^{\infty}\left(\mathbb{R}^{N}\right)$ and $\varepsilon>0$. Since $V \in \mathrm{L}_{\text {loc }}^{\alpha+1}\left(\mathbb{R}^{N}\right)$ by (7.16) and (1.2), we see that

$$
\left|\int_{\{|x|<\varepsilon\}} V\left(\Delta \varphi-\frac{1}{2} \nabla \cdot(x \varphi)+\frac{1}{\alpha} \varphi+|V|^{\alpha} \varphi\right)\right| \leq C \int_{\{|x|<\varepsilon\}}\left(|V|+|V|^{\alpha+1}\right) \underset{\varepsilon \downarrow 0}{\longrightarrow} 0 .
$$

Next, since $f \in \mathrm{C}^{2}(0, \infty)$ is a solution of (1.5), it follows that $V$ satisfies (7.21) in $\mathrm{C}\left(\mathbb{R}^{N} \backslash\{0\}\right)$, so that integration by parts yields

$$
\int_{\{|x|>\varepsilon\}} V\left(\Delta \varphi-\frac{1}{2} \nabla \cdot(x \varphi)+\frac{1}{\alpha} \varphi+|V|^{\alpha} \varphi\right)=-\int_{\{|x|=\varepsilon\}}\left(V \frac{\partial \varphi}{\partial r}-\varphi \frac{\partial V}{\partial r}-\frac{\varepsilon}{2} V \varphi\right) .
$$

On the other hand, $r^{N-1}\left(|f(r)|+\left|f^{\prime}(r)\right|\right) \rightarrow 0$ as $r \downarrow 0$ by (7.16), (7.17) and (1.2). Therefore,

$$
\int_{\{|x|=\varepsilon\}}\left(|V|+\left|\frac{\partial V}{\partial r}\right|\right) \underset{\varepsilon \downarrow 0}{\longrightarrow} 0,
$$


and it follows from (7.22) and (7.23) that

$$
\int_{\mathbb{R}^{N}} V\left(\Delta \varphi-\frac{1}{2} \nabla \cdot(x \varphi)+\frac{1}{\alpha} \varphi+|V|^{\alpha} \varphi\right)=0 .
$$

Since $\varphi \in \mathrm{C}_{\mathrm{c}}^{\infty}\left(\mathbb{R}^{N}\right)$ is arbitrary, we see that $V$ solves (7.21) in $\mathcal{D}^{\prime}\left(\mathbb{R}^{N}\right)$. Properties (i) and (ii) now follows from Lemma 7.1 of [5]. Next, suppose (1.2) and $\ell=\beta^{\frac{1}{\alpha}}$ in (7.16). It follows from Proposition 7.1 with $a=1$ that $\left|r^{\frac{2}{\alpha}} f(r)-\beta^{\frac{1}{\alpha}}\right| \leq C r^{\rho}$ for $r \leq 1$. Moreover, it follows from (7.18) that $\left|r^{\frac{2}{\alpha}} f(r)-\beta^{\frac{1}{\alpha}}\right| \leq C$ for $r \geq 1$; and so,

$$
\left|r^{\frac{2}{\alpha}} f(r)-\beta^{\frac{1}{\alpha}}\right| \leq C\left(\frac{r}{r+1}\right)^{\rho}, \quad r>0
$$

Using (7.19), this implies that

$$
\left.|| x\right|^{\frac{2}{\alpha}} U(t, x)-\beta^{\frac{1}{\alpha}}|=|\left(\frac{|x|}{\sqrt{t}}\right)^{\frac{2}{\alpha}} f\left(\frac{|x|}{\sqrt{t}}\right)-\beta^{\frac{1}{\alpha}} \mid \leq C\left(\frac{|x|}{|x|+\sqrt{t}}\right)^{\rho}
$$

for $t>0, x \neq 0$. This proves Property (iii). Finally, the last statement is proven in Theorem 1.3 of [5].

Proof of Theorem 1.4. Let $f \in \mathrm{C}^{2}(0, \infty)$ be a solution of the equation (1.5) having the singularity $r^{\frac{2}{\alpha}} f(r) \rightarrow \beta^{\frac{1}{\alpha}}$ as $r \rightarrow 0$. It follows from Proposition 7.2 that the limit (1.15) exists and is finite, and that $U$ defined by (1.16) satisfies (1.6), (1.7) (for all $t>0, x \neq 0$ ) and (1.9) with $U_{0}(x)=\mu|x|^{-\frac{2}{\alpha}}$, and that $U$ is a solution of (1.1) in $\mathcal{D}^{\prime}\left((0, T) \times \mathbb{R}^{N}\right)$. The result now follows by applying Theorem 1.1 with $S=1$.

\section{Appendix A. The heat equation with inverse square potential}

In this section, we collect some properties of the homogeneous and nonhomogeneous heat equation with inverse square potential. We assume (1.20), and we consider the operator $H$ defined by (1.21) and the corresponding semigroup $\left(e^{t H}\right)_{t \geq 0}$ on $\mathrm{L}^{2}\left(\mathbb{R}^{N}\right)$. We first recall some well-known properties of $H$.

Lemma A.1. Assume (1.20), and let the operator $H$ on $\mathrm{L}^{2}\left(\mathbb{R}^{N}\right)$ be defined by (1.21).

(i) $H$ is a self-adjoint, negative operator, and $D(H) \hookrightarrow \mathrm{H}^{1}\left(\mathbb{R}^{N}\right)$ where $D(H)$ is equipped with the graph norm.

(ii) $H$ is the generator of a $C_{0}$ semigroup of contractions $\left(e^{t H}\right)_{t \geq 0}$, which is an analytic semigroup on $\mathrm{L}^{2}\left(\mathbb{R}^{N}\right)$.

(iii) Given $u_{0} \in \mathrm{L}^{2}\left(\mathbb{R}^{N}\right), u(t)=e^{t H} u_{0}$ satisfies $u \in \mathrm{C}\left((0, \infty), \mathrm{H}^{1}\left(\mathbb{R}^{N}\right)\right)$ and

$$
\|u(t)\|_{\mathrm{H}^{1}} \leq C\left(1+t^{-\frac{1}{2}}\right)\left\|u_{0}\right\|_{\mathrm{L}^{2}}
$$

for all $t>0$, where the constant $C$ is independent of $u_{0}$.

Proof. Recall that

$$
\left\|\frac{u}{|\cdot|}\right\|_{\mathrm{L}^{2}} \leq \frac{2}{N-2}\|\nabla u\|_{\mathrm{L}^{2}}
$$

and

$$
\left\|\frac{u}{|\cdot|^{2}}\right\|_{L^{\frac{2 N}{N+2}}} \leq C\|\nabla u\|_{\mathrm{L}^{2}}
$$

for all $u \in \mathrm{H}^{1}\left(\mathbb{R}^{N}\right)$. Inequality (A.2) is the standard Hardy estimate, and inequality (A.3) is a CaffarelliKohn-Nirenberg inequality, see inequality (1.4) in [3]. Since $\mathrm{L}^{\frac{2 N}{N+2}}\left(\mathbb{R}^{N}\right) \hookrightarrow \mathrm{H}^{-1}\left(\mathbb{R}^{N}\right)$, it follows from (A.3) 
that

$$
L \in \mathcal{L}\left(\mathrm{H}^{1}\left(\mathbb{R}^{N}\right), \mathrm{H}^{-1}\left(\mathbb{R}^{N}\right)\right) \text {, where } L u=\Delta u+\beta(\alpha+1)|x|^{-2} u .
$$

Note that

$$
L u=H u, \quad u \in D(H)
$$

Since

$$
\langle L u, v\rangle_{\mathrm{H}^{-1}, \mathrm{H}^{1}}=-\int_{\mathbb{R}^{N}} \nabla u \cdot \nabla v+\beta(\alpha+1) \int_{\mathbb{R}^{N}} \frac{u v}{|x|^{2}}
$$

for all $u, v \in \mathrm{H}^{1}\left(\mathbb{R}^{N}\right)$, it follows from (1.20) and (A.2) that

$$
\langle L u, u\rangle_{\mathrm{H}^{-1}, \mathrm{H}^{1}}=-\int_{\mathbb{R}^{N}}|\nabla u|^{2}+\beta(\alpha+1) \int_{\mathbb{R}^{N}} \frac{|u|^{2}}{|x|^{2}} \leq-\nu \int_{\mathbb{R}^{N}}|\nabla u|^{2},
$$

where $\nu=1-\frac{4 \beta(\alpha+1)}{(N-2)^{2}}>0$. We deduce by Lax-Milgram's theorem that the map $u \mapsto-L u+u$ is a homeomorphism $\mathrm{H}^{1}\left(\mathbb{R}^{N}\right) \rightarrow \mathrm{H}^{-1}\left(\mathbb{R}^{N}\right)$. Let now $f \in \mathrm{L}^{2}\left(\mathbb{R}^{N}\right)$. It follows that there exists a unique $u \in \mathrm{H}^{1}\left(\mathbb{R}^{N}\right)$ such that $-L u+u=f$. In particular, $L u=u-f \in \mathrm{L}^{2}\left(\mathbb{R}^{N}\right)$, so that $u \in D(H)$ and $-H u+u=f$. Thus we see that $R(I-H)=\mathrm{L}^{2}\left(\mathbb{R}^{N}\right)$. In addition, given $u, v \in D(H)$, we deduce from (A.5) that

$$
(H u, v)_{\mathrm{L}^{2}}=\langle L u, v\rangle_{\mathrm{H}^{-1}, \mathrm{H}^{1}}=\langle L v, u\rangle_{\mathrm{H}^{-1}, \mathrm{H}^{1}}=(H v, u)_{\mathrm{L}^{2}} .
$$

Thus we see that $H$ is symmetric. Moreover, it follows from (A.6) that

$$
(H u, u)_{\mathrm{L}^{2}} \leq 0,
$$

for all $u \in D(H)$. Therefore $H \leq 0$ and $R(I-H)=\mathrm{L}^{2}\left(\mathbb{R}^{N}\right)$, so that $H$ is $m$-dissipative. Since $H$ is symmetric, we conclude that $H$ is a negative self-adjoint operator (see e.g. [2], Prop. 7.6, p. 193). In addition, it follows from (A.6) that

$$
\|u\|_{\mathrm{H}^{1}}^{2}=\|u\|_{\mathrm{L}^{2}}^{2}+\|\nabla u\|_{\mathrm{L}^{2}}^{2} \leq\left(1+\frac{1}{\nu}\right)\left(\|u\|_{\mathrm{L}^{2}}^{2}-(H u, u)_{\mathrm{L}^{2}}\right) \leq\left(1+\frac{1}{\nu}\right)\|u\|_{\mathrm{L}^{2}}\|u\|_{D(H)},
$$

so that $D(H) \hookrightarrow \mathrm{H}^{1}\left(\mathbb{R}^{N}\right)$. This proves (i), and Property (ii) follows from the standard semigroup theory, see e.g. [8], Chapter IX, Theorem 1.24 and Example 1.25.

Next, let $u_{0} \in \mathrm{C}_{\mathrm{c}}^{\infty}\left(\mathbb{R}^{N}\right)$, so that $u_{0} \in D(H)$, and let $u(t)=e^{t H} u_{0}$, so that $u \in \mathrm{C}([0, \infty), D(H)) \cap$ $\mathrm{C}^{1}\left([0, \infty), \mathrm{L}^{2}\left(\mathbb{R}^{N}\right)\right) \cap \mathrm{C}^{\infty}((0, \infty), D(H))$ and $\partial_{t} u=H u$. Multiplying the equation by $-H u$, we obtain

$$
\frac{\mathrm{d}}{d t}(-H u, u)_{\mathrm{L}^{2}}=-\|H u\|_{\mathrm{L}^{2}}^{2} \leq 0
$$

so that

$$
(-H u(t), u(t))_{\mathrm{L}^{2}} \leq(-H u(s), u(s))_{\mathrm{L}^{2}}, \quad 0 \leq s \leq t .
$$


Next, multiplying the equation by $u$ and integrating on $(0, t) \times \mathbb{R}^{N}$,

$$
\frac{1}{2}\|u(t)\|_{\mathrm{L}^{2}}^{2}+\int_{0}^{t}(-H u(s), u(s))_{\mathrm{L}^{2}} \mathrm{~d} s=\frac{1}{2}\left\|u_{0}\right\|_{\mathrm{L}^{2}}^{2},
$$

so that by (A.7)

$$
2 t(-H u(t), u(t))_{\mathrm{L}^{2}} \leq\left\|u_{0}\right\|_{\mathrm{L}^{2}}^{2} .
$$

Using (A.6) and the fact that $\left(e^{t H}\right)_{t \geq 0}$ is a semigroup of contractions on $\mathrm{L}^{2}\left(\mathbb{R}^{N}\right)$, we conclude that (A.1) holds. Since $\mathrm{C}_{\mathrm{c}}^{\infty}\left(\mathbb{R}^{N}\right)$ is dense in $\mathrm{L}^{2}\left(\mathbb{R}^{N}\right)$, Property (iii) follows by a standard density argument.

Lemma A.2. Given any $w_{0} \in \mathrm{L}^{2}\left(\mathbb{R}^{N}\right), w(t)=: e^{t H} w_{0}$ satisfies $w(t) \rightarrow w_{0}$ in $\mathrm{L}^{2}\left(\mathbb{R}^{N}\right)$ as $t \rightarrow 0$, and $w$ is a solution of

$$
\partial_{t} w=\Delta w+\beta(\alpha+1)|x|^{-2} w
$$

in $\mathrm{C}\left((0, T), \mathrm{L}^{2}\left(\mathbb{R}^{N}\right)\right)$, and in particular in $\mathcal{D}^{\prime}\left((0, \infty) \times \mathbb{R}^{N}\right)$.

Proof. That $w(t) \rightarrow w_{0}$ in $\mathrm{L}^{2}\left(\mathbb{R}^{N}\right)$ as $t \rightarrow 0$ follows from the fact that $\left(e^{t H}\right)_{t \geq 0}$ is a $C_{0}$ semigroup on $\mathrm{L}^{2}\left(\mathbb{R}^{N}\right)$. That the equation (A.8) is satisfied in $\mathrm{C}\left((0, T), \mathrm{L}^{2}\left(\mathbb{R}^{N}\right)\right)$ follows from the analyticity of the semigroup $\left(e^{t H}\right)_{t \geq 0}$ on $\mathrm{L}^{2}\left(\mathbb{R}^{N}\right)$.

Lemma A.3. There exists $\varsigma>0$ such that

$$
\left\|\frac{1}{h(t, x)} \int_{\mathbb{R}^{N}} \mathcal{K}(t, x, y) 1_{\{|y|>n\}} w_{0}(y) \mathrm{d} y\right\|_{L^{\infty}\left(|x| \leq \frac{n}{2}\right)} \leq\left(1+\frac{\sqrt{t}}{n}\right)^{\eta} e^{-\varsigma \frac{n^{2}}{t}}\left\|w_{0}\right\|_{\mathrm{L}^{\infty}}
$$

for all $t>0$ and all $w_{0} \in \mathrm{L}^{\infty}\left(\mathbb{R}^{N}\right)$.

Proof. It follows from (1.22) and (1.23) that

$$
\begin{aligned}
\frac{1}{h(t, x)} \int_{\mathbb{R}^{N}} \mathcal{K}(t, x, y) 1_{\{|y|>n\}}\left|w_{0}\right| \mathrm{d} y & \leq A\left\|w_{0}\right\|_{L^{\infty}} t^{-\frac{N}{2}} \int_{|y|>n} e^{-\frac{|x-y|^{2}}{a t}} h(t, y) \mathrm{d} y \\
& \leq\left(1+\frac{\sqrt{t}}{n}\right)^{\eta} A\left\|w_{0}\right\|_{L^{\infty}} t^{-\frac{N}{2}} \int_{|y|>n} e^{-\frac{|x-y|^{2}}{a t}} \mathrm{~d} y \\
& =\left(1+\frac{\sqrt{t}}{n}\right)^{\eta}\left(\frac{4 \pi}{a}\right)^{\frac{N}{2}} A\left\|w_{0}\right\|_{L^{\infty}} e^{\frac{a t}{4} \Delta} 1_{\{|y|>n\}} .
\end{aligned}
$$

Setting $z=\frac{x}{n}$ and $\tau=\frac{t}{n^{2}}$, we have

$$
\left(e^{t \Delta} 1_{\{|y|>n\}}\right)(x)=\left(e^{\tau \Delta} 1_{\{|y|>1\}}\right)(z)=(4 \pi \tau)^{-\frac{N}{2}} \int_{|y|>1} e^{-\frac{|z-y|^{2}}{4 \tau}} .
$$

If $|x| \leq \frac{n}{2}$ then $|z| \leq \frac{1}{2}$. For $|y| \geq 1$ we have $|z-y| \geq|y|-|z| \geq \frac{|y|}{2}$. Thus we see that for $|x| \leq \frac{n}{2}$

$$
(4 \pi)^{\frac{N}{2}}\left(e^{t \Delta} 1_{\{|y|>n\}}\right)(x)=\tau^{-\frac{N}{2}} \int_{|y|>1} e^{-\frac{|z-y|^{2}}{4 \tau}} \leq \tau^{-\frac{N}{2}} \int_{|y|>1} e^{-\frac{|y|^{2}}{16 \tau}} \leq e^{-\frac{\varsigma}{\tau}}=e^{-\frac{\varsigma n^{2}}{t}}
$$

for all $t>0$, where $\varsigma>0$. Hence the result follows. 
Lemma A.4. For every $t>0$, the operator $e^{t H}$ can be extended to a continuous operator $\mathrm{L}^{\infty}\left(\mathbb{R}^{N}\right) \rightarrow$ $\mathrm{L}^{\frac{2 N}{N-2}}\left(\mathbb{R}^{N}\right)+\mathrm{L}^{\infty}\left(\mathbb{R}^{N}\right)$. Moreover, for every $w_{0} \in \mathrm{L}^{\infty}\left(\mathbb{R}^{N}\right), w(t)=e^{t H} w_{0}$ satisfies the equation (A.8) in $\mathcal{D}^{\prime}\left((0, \infty) \times \mathbb{R}^{N}\right)$, and

$$
|w(t, x)| \leq C\left\|w_{0}\right\|_{\mathrm{L}^{\infty}} h(t, x)
$$

In addition, $e^{t H} w_{0} \rightarrow w_{0}$ in $\mathrm{L}_{\mathrm{loc}}^{1}\left(\mathbb{R}^{N}\right)$ as $t \rightarrow 0$.

Proof. Let $w_{0} \in \mathrm{L}^{\infty}\left(\mathbb{R}^{N}\right)$. Set $w_{0}^{n}=1_{\{|x|<n\}} w_{0} \in \mathrm{L}^{2}\left(\mathbb{R}^{N}\right)$ and $w_{n}(t)=e^{t H} w_{0}^{n}$. It follows from Lemma A.3 that for $m \geq n$

$$
\frac{1}{h}\left|w_{n}(t)-w_{m}(t)\right| \leq \frac{1}{h} \int_{\mathbb{R}^{N}} \mathcal{K}(t, x, y)\left|1_{\{n<|x|<m\}} w_{0}\right| \mathrm{d} y \leq \frac{1}{h} \int_{\mathbb{R}^{N}} \mathcal{K}(t, x, y) 1_{\{|x|>n\}}\left|w_{0}\right| \mathrm{d} y \underset{n \rightarrow \infty}{\longrightarrow} 0
$$

in $\mathrm{L}^{\infty}((0, T) \times\{|x|<T\})$ for every $T>0$. Since $h \in \mathrm{L}^{\infty}\left((0, T), \mathrm{L}^{\frac{2 N}{N-2}}(\{|x|<T\})\right)$, we conclude easily that there exists $w \in \mathrm{L}_{\text {loc }}^{\frac{2 N}{N-2}}\left((0, \infty) \times \mathbb{R}^{N}\right)$ such that $w_{n} \rightarrow w$ as $n \rightarrow \infty$ in $\mathrm{L}^{\infty}\left((0, T), \mathrm{L}^{\frac{2 N}{N-2}}(\{|x|<T\})\right)$ for every $T>0$. Moreover, since $w_{n}(t) \rightarrow 1_{\{|x|<n\}} w_{0}$ in $\mathrm{L}^{2}\left(\mathbb{R}^{N}\right)$ as $t \rightarrow 0$, by Lemma A.2, we deduce that $w(t) \rightarrow w_{0}$ in $\mathrm{L}_{\text {loc }}^{1}\left(\mathbb{R}^{N}\right)$ as $t \rightarrow 0$. Finally, it follows from (1.22) that

$$
\left|w_{n}(t)\right| \leq(4 \pi)^{\frac{N}{2}} h(t) A e^{\frac{a t}{4} \Delta}\left(h(t)\left|1_{\{|x|<n\}} w_{0}\right|\right) \leq C\left\|w_{0}\right\|_{L^{\infty}} h(t) e^{\frac{a t}{4} \Delta}(h(t)) .
$$

Note that $h(t, y) \leq C\left(1+t^{\frac{\eta}{2}}|x|^{-\eta}\right)$. Moreover, since $e^{\frac{a t}{4} \Delta}\left(|x|^{-\eta}\right) \leq C\left(t+|x|^{2}\right)^{-\eta} \leq C t^{-\frac{\eta}{2}}$ by ([4], Cor. 8.3), we see that $e^{\frac{a t}{4} \Delta}(h(t)) \leq C$ and (A.9) follows.

Let now $\theta \in \mathrm{C}_{\mathrm{c}}^{\infty}\left((0, \infty) \times \mathbb{R}^{N}\right)$ and let $T>0$ be sufficiently large so that

$$
\operatorname{supp} \theta \subset(0, T) \times\{|x|<T\} .
$$

Since $w_{0}^{n} \in \mathrm{L}^{2}\left(\mathbb{R}^{N}\right)$, it follows from Lemma A.2 that $w_{n}$ is a solution of $\left(\right.$ A.8) in $\mathcal{D}^{\prime}\left((0, \infty) \times \mathbb{R}^{N}\right)$; and so,

$$
\begin{aligned}
\int_{0}^{\infty} \int_{\mathbb{R}^{N}} w\left(-\partial_{t} \theta-\Delta \theta-|x|^{-2} \theta\right)= & \int_{0}^{\infty} \int_{\mathbb{R}^{N}} w_{n}\left(-\partial_{t} \theta-\Delta \theta-|x|^{-2} \theta\right) \\
& +\int_{0}^{\infty} \int_{\mathbb{R}^{N}}\left(w-w_{n}\right)\left(-\partial_{t} \theta-\Delta \theta-|x|^{-2} \theta\right) \\
= & \int_{0}^{\infty} \int_{\mathbb{R}^{N}}\left(w-w_{n}\right)\left(-\partial_{t} \theta-\Delta \theta-|x|^{-2} \theta\right)
\end{aligned}
$$

On the other hand, $h \in \mathrm{L}^{1}((0, T) \times\{|x|<T\}$, so it follows from (A.10) and (A.11) that

$$
\int_{0}^{T} \int_{\mathbb{R}^{N}}\left(w-w_{n}\right)\left(-\partial_{t} \theta-\Delta \theta-|x|^{-2} \theta\right) \underset{n \rightarrow \infty}{\longrightarrow} 0 .
$$

Therefore,

$$
\int_{0}^{\infty} \int_{\mathbb{R}^{N}} w\left(-\partial_{t} \theta-\Delta \theta-|x|^{-2} \theta\right)=0
$$

showing that $w$ satisfies $\left(\right.$ A.8) in $\mathcal{D}^{\prime}\left((0, \infty) \times \mathbb{R}^{N}\right)$. 
Lemma A.5. Let $T>0, f \in \mathrm{L}^{\infty}\left((0, T), \mathrm{L}^{2}\left(\mathbb{R}^{N}\right)\right)$, and set

$$
w(t)=\int_{0}^{t} e^{(t-s) H} f(s) \mathrm{d} s,
$$

for $0 \leq t \leq T$. It follows that $w \in \mathrm{C}\left([0, T], \mathrm{H}^{1}\left(\mathbb{R}^{N}\right)\right) \cap \mathrm{W}^{1, \infty}\left((0, T), \mathrm{H}^{-1}\left(\mathbb{R}^{N}\right)\right)$ and that

$$
\partial_{t} w-\Delta w-\beta(\alpha+1)|x|^{-2} w=f,
$$

in $\mathrm{L}^{\infty}\left((0, T), \mathrm{H}^{-1}\left(\mathbb{R}^{N}\right)\right)$, and in particular in $\mathcal{D}^{\prime}\left((0, T) \times \mathbb{R}^{N}\right)$.

Proof. Suppose first $f \in \mathrm{C}_{\mathrm{c}}^{\infty}\left((0, T) \times\left(\mathbb{R}^{N} \backslash\{0\}\right)\right)$. It follows in particular that $f \in \mathrm{C}([0, T], D(H))$ so that $w \in \mathrm{C}([0, T], D(H)) \cap \mathrm{C}^{1}\left([0, T], \mathrm{L}^{2}\left(\mathbb{R}^{N}\right)\right)$ and equation (A.13) holds in $\mathrm{C}\left([0, T], \mathrm{L}^{2}\left(\mathbb{R}^{N}\right)\right)$, see e.g. [14], Chapter 4, Corollary 2.6. In particular, $w \in \mathrm{C}\left([0, T], \mathrm{H}^{1}\left(\mathbb{R}^{N}\right)\right)$ and, using (A.1),

$$
\|w\|_{\mathrm{L}^{\infty}\left((0, T), \mathrm{H}^{1}\left(\mathbb{R}^{N}\right)\right)} \leq C \int_{0}^{t}\left(1+(t-s)^{-\frac{1}{2}}\right)\|f(s)\|_{\mathrm{L}^{2}} \mathrm{~d} s \leq C\|f\|_{\mathrm{L}^{4}\left((0, T), \mathrm{L}^{2}\left(\mathbb{R}^{N}\right)\right)} .
$$

Let now $f \in \mathrm{L}^{\infty}\left((0, T), \mathrm{L}^{2}\left(\mathbb{R}^{N}\right)\right)$ and $\left(f_{n}\right)_{n \geq 1} \subset \mathrm{C}_{\mathrm{c}}^{\infty}\left((0, T) \times\left(\mathbb{R}^{N} \backslash\{0\}\right)\right)$ such that $f_{n} \rightarrow f$ in $\mathrm{L}^{4}\left((0, T), \mathrm{L}^{2}\left(\mathbb{R}^{N}\right)\right)$. Let $w_{n}$ be given by (A.12) with $f$ replaced by $f_{n}$. It follows that $w_{n} \rightarrow w$ in $\mathrm{C}\left([0, T], \mathrm{L}^{2}\left(\mathbb{R}^{N}\right)\right)$. Moreover, we deduce from (A.14) with $f$ replaced by $f_{n}-f_{m}$ that $w_{n}$ is a Cauchy sequence in $\mathrm{C}\left([0, T], \mathrm{H}^{1}\left(\mathbb{R}^{N}\right)\right)$. Therefore, $w \in \mathrm{C}\left([0, T], \mathrm{H}^{1}\left(\mathbb{R}^{N}\right)\right)$ and $w_{n} \rightarrow w$ in $\mathrm{C}\left([0, T], \mathrm{H}^{1}\left(\mathbb{R}^{N}\right)\right)$. Using (A.4), we deduce that

$$
\Delta w_{n}+\beta(\alpha+1)|x|^{-2} w_{n} \rightarrow \Delta w+\beta(\alpha+1)|x|^{-2} w,
$$

in $\mathrm{C}\left([0, T], \mathrm{H}^{-1}\left(\mathbb{R}^{N}\right)\right)$. Since $\partial_{t} w_{n}=H w_{n}+f_{n}$, we conclude that

$$
\partial_{t} w_{n} \underset{n \rightarrow \infty}{\longrightarrow} \Delta w+\beta(\alpha+1)|x|^{-2} w+f
$$

in $\mathrm{L}^{4}\left((0, T), \mathrm{H}^{-1}\left(\mathbb{R}^{N}\right)\right)$. On the other hand, $\partial_{t} w_{n} \rightarrow \partial_{t} w$ in $\mathcal{D}^{\prime}\left((0, T), H^{1}\left(\mathbb{R}^{N}\right)\right)$, so that (A.13) holds in $\mathrm{L}^{4}\left((0, T), \mathrm{H}^{-1}\left(\mathbb{R}^{N}\right)\right)$. Since all terms in (A.13), except perhaps $\partial_{t} w$, belong to $\mathrm{L}^{\infty}\left((0, T), \mathrm{H}^{-1}\left(\mathbb{R}^{N}\right)\right)$, we see that $\partial_{t} w \in \mathrm{L}^{\infty}\left((0, T), \mathrm{H}^{-1}\left(\mathbb{R}^{N}\right)\right)$ and that (A.13) holds in $\mathrm{L}^{\infty}\left((0, T), \mathrm{H}^{-1}\left(\mathbb{R}^{N}\right)\right)$.

Lemma A.6. Let $T>0, \alpha>0$ and $u, f \in \mathrm{L}_{\mathrm{loc}}^{\infty}\left((0, T) \times\left(\mathbb{R}^{N} \backslash\{0\}\right)\right)$ satisfy

$$
\partial_{t} u-\Delta u=f
$$

in $\mathcal{D}^{\prime}\left((0, T) \times\left(\mathbb{R}^{N} \backslash\{0\}\right)\right)$. It follows that $u \in \mathrm{C}\left((0, T) \times\left(\mathbb{R}^{N} \backslash\{0\}\right)\right)$.

Proof. This is standard interior parabolic regularity. For any $0<\delta<\min \left\{1, \frac{T}{2}\right\}$, we define $\mathcal{O}_{\delta}=(\delta, T-\delta) \times\{x \in$ $\left.\mathbb{R}^{N} ; \delta<|x|<\frac{1}{\delta}\right\}$. Fix $p \in(N+1, \infty)$, so that $\mathrm{W}^{1, p}\left(\mathcal{O}_{\delta}\right) \hookrightarrow \mathrm{C}\left(\overline{\mathcal{O}_{\delta}}\right)$. Let $\left(\rho_{n}\right)_{n \geq 1}$ be a regularizing sequence with $\operatorname{supp} \rho_{n} \subset\left\{\left(t^{2}+|x|^{2}\right)^{\frac{1}{2}}<\frac{1}{n}\right\}$. Fix $0<\varepsilon<\min \left\{\frac{1}{2}, \frac{T}{4}\right\}$. It follows that for $n \geq \frac{1}{\varepsilon}, u_{n}=: \rho_{n} \star u$ and $f_{n}=: \rho_{n} \star f$, where the convolution is in $\mathbb{R}^{1+N}$, are well defined on $\mathcal{O}_{\varepsilon}$, and that $u_{n} \rightarrow u$ and $f_{n} \rightarrow f$ in $\mathrm{L}^{p}\left(\mathcal{O}_{\varepsilon}\right)$ as $n \rightarrow \infty$. Moreover, $u_{n}, f_{n} \in \mathrm{C}^{\infty}\left(\overline{\mathcal{O}_{\varepsilon}}\right)$ and $\partial_{t} u_{n}-\Delta u_{n}=f_{n}$ in $\mathcal{O}_{\varepsilon}$. By parabolic interior regularity (see e.g. [9], Thm. 7.22),

$$
\begin{aligned}
\left\|u_{n}-u_{m}\right\|_{\mathrm{L}^{\infty}\left(\mathcal{O}_{2 \varepsilon}\right)} & \leq C\left\|u_{n}-u_{m}\right\|_{\mathrm{W}^{1, p}\left(\mathcal{O}_{2 \varepsilon}\right)} \\
& \leq C\left(\left\|f_{n}-f_{m}\right\|_{\mathrm{L}^{p}\left(\mathcal{O}_{\varepsilon}\right)}+\left\|u_{n}-u_{m}\right\|_{\mathrm{L}^{p}\left(\mathcal{O}_{\varepsilon}\right)}\right) \underset{n, m \rightarrow \infty}{\longrightarrow} 0 .
\end{aligned}
$$

Therefore $u_{n}$ is a Cauchy sequence in $\mathrm{L}^{\infty}\left(\mathcal{O}_{2 \varepsilon}\right)$, so that $u \in \mathrm{C}\left(\overline{\mathcal{O}_{2 \varepsilon}}\right)$. The result follows by letting $\varepsilon \rightarrow 0$. 


\section{REFERENCES}

[1] P. Baras and J. Goldstein, The heat equation with a singular potential. Trans. Am. Math. Soc. 284 (1984) $121-139$.

[2] H. Brezis, Functional analysis, Sobolev spaces and partial differential equations. Universitext. Springer, New York (2011).

[3] L. Caffarelli, R.V. Kohn and L. Nirenberg, First order interpolation inequalities with weights. Compositio Math. 53 (1984) 259-275.

[4] T. Cazenave, F. Dickstein, M. Escobedo and F.B. Weissler, Self-similar solutions of a nonlinear heat equation. J. Math. Sci. Univ. Tokyo 8 (2001) 501-540.

[5] T. Cazenave, F. Dickstein, I. Naumkin and F.B. Weissler, Sign-changing self-similar solutions of the nonlinear heat equation with positive initial value. Am. J. Math. 142 (2020), 1439-1495.

[6] T. Cazenave, F. Dickstein, I. Naumkin and F.B. Weissler, Perturbations of self-similar solutions. Dyn. Partial Differ. Equ. 16 (2019) 151-183.

[7] M. Hoshino and E. Yanagida, Convergence rate to singular steady states in a semilinear parabolic equation. Nonlinear Anal. 131 (2016) 98-111.

[8] T. Kato, Perturbation theory for linear operators, Reprint of the 1980 edition. Classics in Mathematics. Springer-Verlag, Berlin (1995).

[9] G.M. Lieberman, Second order parabolic differential equations. World Scientific Publishing Co. Inc. River Edge, NJ (1996).

[10] V.A. Liskevich and Z. Sobol, Estimates of integral kernels for semigroups associated with second-order elliptic operators with singular coefficients. Potential Anal. 18 (2003) 359-390.

[11] R. Mazzeo and F. Pacard, A construction of singular solutions for a semilinear elliptic equation using asymptotic analysis. $J$. Differential Geom. 44 (1996) 331-370.

[12] P.D. Milman and Yu. A. Semenov, Heat kernel bounds and desingularizing weights. J. Funct. Anal. 202 (2003) 1-24.

[13] L. Moschini and A. Tesei, Parabolic Harnack inequality for the heat equation with inverse-square potential. Forum Math. 19 (2007) 407-427.

[14] A. Pazy, Semi-groups of linear operators and applications to partial differential equations. Appl. Math. Sci. 44 (1983).

[15] P. Quittner and P. Souplet, Superlinear parabolic problems. Blow-up, global existence and steady states. Second edition. Birkhäuser Advanced Texts: Basler Lehrbücher. [Birkhäuser Advanced Texts: Basel Textbooks]. Birkhäuser/Springer, Cham (2019).

[16] S. Sato, Blow-up at space infinity of a solution with a moving singularity for a semilinear parabolic equation. Commun. Pure Appl. Anal. 10 (2011) 1225-1237.

[17] S. Sato and E. Yanagida, Solutions with moving singularities for a semilinear parabolic equation. J. Differ. Equ. 246 (2009) $724-748$.

[18] S. Sato and E. Yanagida, Forward self-similar solution with a moving singularity for a semilinear parabolic equation. Discrete Contin. Dynam. Syst. 26 (2010) 1313-1331.

[19] S. Sato and E. Yanagida, Appearance of anomalous singularities in a semilinear parabolic equation. Commun. Pure Appl. Anal. 11 (2012) 387-405.

[20] S. Sato and E. Yanagida, Asymptotic behavior of singular solutions for a semilinear parabolic equation. Discrete Contin. Dyn. Syst. 32 (2012) 4027-4043.

[21] J. Serrin and H. Zou, Classification of positive solutions of quasilinear elliptic equations. Topol. Methods Nonlinear Anal. 3 (1994) 1-25.

[22] P. Souplet and F.B. Weissler, F.B.: Regular self-similar solutions to the nonlinear heat equation with initial data above the singular steady state. Ann. Inst. Henri Poincaré Anal. Non Linéaire 20 (2003) 213-235.

[23] J.L. Vázquez and E. Vázquez, The Hardy inequality and the asymptotic behaviour of the heat equation with an inverse-square potential. J. Funct. Anal. 173 (2000) 103-153. 